\title{
Self-Assembled Networks of Short and Long Chitin Nanoparticles for Oil/Water Interfacial Superstabilization
}

\author{
Long Bai, ${ }^{*} \dagger$ Siqi Huan, ${ }^{\dagger}$ Wenchao Xiang, ${ }^{\dagger}$ Liang Liu, ${ }^{\dagger}{ }^{\dagger}$ Yang Yang, ${ }^{\S}$ Robertus Wahyu N. Nugroho,
} Yimin Fan, ${ }^{+\infty}$ and Orlando J. Rojas, ${ }^{* \dagger+}$

${ }^{\dagger}$ Bio-Based Colloids and Materials, Department of Bioproducts and Biosystems, Aalto University, Vuorimiehentie1, Espoo 02150, Finland

${ }^{\ddagger}$ Jiangsu Co-Innovation Center of Efficient Processing and Utilization of Forest Resources, Jiangsu Key Lab of Biomass-Based Green Fuel and Chemicals, College of Chemical Engineering, Nanjing Forestry University, 159 Longpan Road, Nanjing 210037, China

${ }^{\S}$ Research Programs Unit, Molecular Neurology, University of Helsinki, Fabianinkatu 33, Helsinki 00014, Finland

Supporting Information
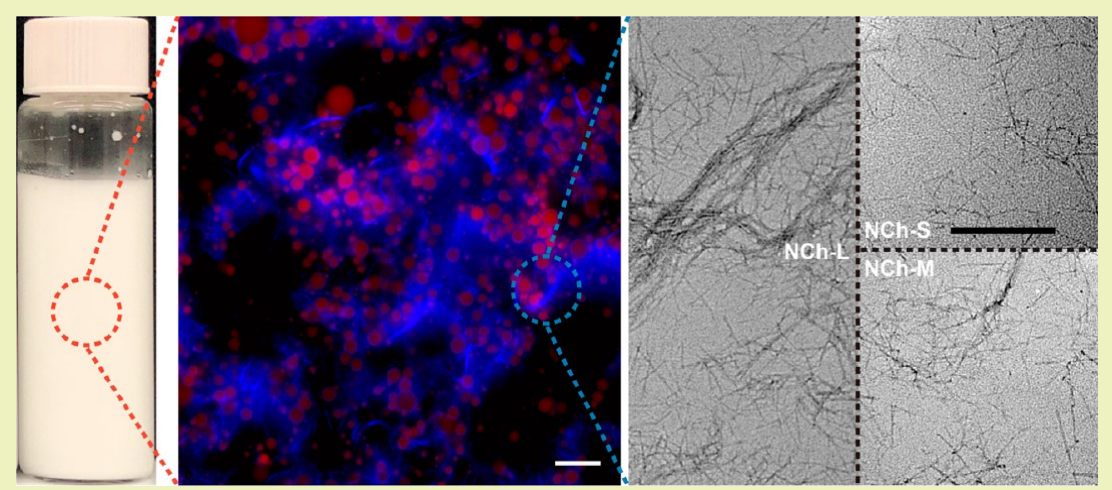

ABSTRACT: Highly charged (zeta potential $\zeta=+105 \mathrm{mV}$, acetate counterions) chitin nanoparticles (NCh) of three different average aspect ratios $(\sim 5,25$, and $>60)$ were obtained by low-energy deconstruction of partially deacetylated chitin. The nanoparticles were effective in reducing the interfacial tension and stabilized the oil/water interface via network formation (interfacial dilatational rheology data) becoming effective in stabilizing Pickering systems, depending on NCh size, composition, and formulation variables. The improved interfacial wettability and electrosteric repulsion facilitated control over the nanoparticle's surface coverage on the oil droplets, their aspect ratio and stability against coalescence during long-term storage. Emulsion superstabilization (oil fractions below 0.5 ) occurred by the microstructuring and thickening effect of NCh that formed networks at concentrations as low as $0.0005 \mathrm{wt} \%$. The ultrasound energy used during emulsion preparation simultaneously reduced the longer nanoparticles, producing very stable, fine oil droplets (diameter $\sim 1 \mu \mathrm{m}$ ). Our findings indicate that NCh surpasses any reported biobased nanoparticle, including nanocelluloses, for its ability to stabilize interfaces at ultralow concentrations and represent a step-forward in efforts to fully replace surfactants in multiphase systems.

KEYWORDS: Nanochitin, Superstabilization, Pickering emulsion, Rodlike particles, Deconstruction

\section{INTRODUCTION}

Oil-in-water emulsions are thermodynamic unstable systems but of great practical interest due to their applicability in foodstuff, cosmetics, and pharmaceutical industries. ${ }^{1}$ In practice, it is the kinetic stability that allows the design of emulsions to overcome their tendency to otherwise phaseseparate, ensuring long shelf life. ${ }^{2}$ Early reports have demonstrated that stable emulsions can be obtained not only by amphiphilic surfactants but also by solid particles of colloidal dimensions, the so-called Pickering systems. ${ }^{3,4} \mathrm{~A}$ strong mechanical barrier at the oil-water interface can be readily generated, preventing droplet breakage, if solid particles are used under a balanced interfacial wettability. ${ }^{5}$ Compared to conventional surfactants, Pickering emulsions offer superior stability at relative low particle loading due to their irreversible adsorption at interfaces. ${ }^{6}$ This feature is highly desirable for environmental-friendly emulsion products because it can minimize the amount of stabilizer used. ${ }^{7}$ Pickering systems are therefore promising in designing and optimizing the formulation of safe and green emulsions.

There is a growing interest to reduce synthetic surfactants and to adopt more biologically sustainable alternatives. In this context, biomass-based particles that are often biodegradable ${ }^{8}$ are ideal candidates. A variety of colloidal particles displaying

Received: August 14, 2018

Revised: January 31, 2019

Published: March 3, 2019 
different size, shape, and surface chemistry have been applied to stabilize Pickering emulsions, including modified cellulose, starches, ${ }^{10}$ and proteins, ${ }^{11}$ among others. Mineral particles, such as those from silica have been considered more widely. ${ }^{12}$ However, fairly few of them can be directly applied in green emulsions due to the chemical treatment needed to tailor their interfacial activity. ${ }^{13}$

The ongoing development of naturally derived, green Pickering stabilizers ${ }^{14}$ has recently began to consider chitin, an insoluble polymer of $\mathrm{N}$-acetylglucosamine ${ }^{15}$ with attributes that include abundance, nontoxicity, biocompatibility, and biodegradability, ${ }^{16,17}$ all of which are added to their physical and mechanical strength. ${ }^{18}$ Besides chitin regenerated from solvents, ${ }^{19,20}$ individual nanofibril-like chitin, which is originally assembled via strong hydrogen bonds, can be easily isolated from the native chitin structure, ${ }^{21}$ offering an opportunity as biobased cationic Pickering stabilizer. The isolation of chitin nanoparticles can proceed by (i) acid hydrolysis, ${ }^{22}$ resulting in highly ordered chitin nanocrystals (ChNC), or (ii) mechanical shearing, ${ }^{23}$ generating rodlike chitin nanoparticles (nanochitin, $\mathrm{NCh}$ ) that retain disordered domains in the structure. Since the length of chitin nanoparticles obtained from both approaches can be somewhat similar, the main difference between the two lies in their degree of crystallinity. ${ }^{24}$ Biliaderis et al. utilized ChNC to stabilize oilin-water Pickering emulsions ${ }^{25}$ and discussed the synergies between interfacial adsorption and aqueous phase networking induced by the nanoparticles. ${ }^{26}$ Pickering emulsions stabilized by $\mathrm{ChNC}$ have been applied as a template to produce biobased products, extending the functional performance of chitin nanomaterials. ${ }^{27,28}$ However, the large oil droplets reported in these ChNC-stabilized Pickering emulsions limit their applicability, ${ }^{29}$ especially when long-term stability is demanded. This is because gravitational separation (creaming to oiling off) occurs more rapidly in systems of large sizes of the dispersed phase. ${ }^{30}$ Overall, the insufficient interfacial wettability of $\mathrm{ChNC}$ reported so far is a major challenge for related Pickering systems to find a real use.

Compared with ChNC obtained from direct acid hydrolysis, a two-step method that includes mechanical disintegration has been developed to produce nanochitin $(\mathrm{NCh}) .{ }^{31}$ Here, partial deacetylation of chitin is necessary before subjecting the system to mechanical shearing. The development in acidic condition of cationic groups naturally present in the deacetylated chitin facilitates its deconstruction by electrostatic repulsion. ${ }^{32}$ A similar principle is used widely in the production of cellulose nanofibrils (CNF) by several oxidative methods that install carboxylic and other groups on the surface of the precursor cellulosic material. Presumably, a random distribution of surface hydrophobic $\mathrm{N}$-acetyl groups in NCh occurs due to the nonselective or site-specific partial deacetylation of chitin. The result is a material, NCh, that displays improved interfacial wettability at the oil-water interface, which is favorable for Pickering stabilization. ${ }^{33}$ Therefore, NCh can be considered as a better chitin-based Pickering stabilizer compared to the commonly reported ChNC. ${ }^{34,35}$ One recent example shows that NCh could stabilize Pickering emulsion but TEMPO-mediated zwitterionical modification of NCh was required, undermining its nature as green stabilizer. ${ }^{36}$

An interesting feature of $\mathrm{NCh}$ is that their characteristic length or axial ratio can be tuned by adjusting the preparation conditions, ${ }^{37}$ which opens the possibility to controllably optimize the properties of Pickering systems. Such effect of nanofibril length on emulsion stability has been explored in the case of nanocelluloses ${ }^{38}$ but not in the case of nanochitin. Herein, a series of nanochitin, displaying varying axial ratio, was produced from ultrasonication and microfluidization. They were used in the formulation of oil-in-water Pickering emulsions, demonstrating a remarkably high efficiency in adjusting droplet size and storage stability. We hypothesize that the secondary disintegration of high-aspect nanochitin is induced during ultrasonicating, which is effective in the production of emulsion droplets by shear. Thus, stable oil-inwater Pickering emulsions are possible at extremely low loadings (as low as $0.001 \mathrm{wt} \% \mathrm{NCh}$ in the aqueous phase or 0.0005 wt $\%$ based on the total mass), a most desirable feature in related systems. This is the first attempt to use unmodified, mechanically isolated chitin nanoparticles as superstabilizer of Pickering emulsions. Moreover, the tailorable properties of nanochitins (axial ratio, charge, and wettability) offer an unsurpassed possibility, compared to any natural system known for application in the stabilization of multiphase systems. Most importantly, a wide range of compositions for high resistance against oiling-off at low stabilizer loadings is demonstrated. Overall, we offer an alternative for the formulation and synthesis of particle-stabilized emulsion that can be used as core component in green systems. The longterm goal of this research is to combine functional attributes of chitin nanoparticles into high-quality, multifunctional Pickering systems, simultaneously endowing antioxidant and antimicrobial capabilities.

\section{EXPERIMENTAL SECTION}

Materials. Alpha $(\alpha)$-chitin was purified from fresh crabs (Callinectes sapidus) that were acquired in the local market (Helsinki harbor, Finland). The purification steps were modified from our previous work. ${ }^{39}$ Briefly, crab shells were pretreated by alternating 1 $\mathrm{M} \mathrm{HCl}$ and $1 \mathrm{M} \mathrm{NaOH}$ immersion for $24 \mathrm{~h}$ of each step with at least three cycles. The obtained residual solid was decolorized by treating with $0.5 \mathrm{wt} \% \mathrm{NaClO}_{2}$ solution ( $\mathrm{pH} 5.0$, acetic acid) for $2 \mathrm{~h}$ at $70^{\circ} \mathrm{C}$. The purified flake-like chitin residues were fully washed with distilled water before crushing into small pieces with a household blender. Purified chitin was stored at $4{ }^{\circ} \mathrm{C}$ for further use. $\mathrm{NaOH}, \mathrm{HCl}$, $\mathrm{NaClO}_{2}, 100 \%$ acetic acid, Nile red, Calcofluor white stain, and dodecane were purchased from Sigma-Aldrich (Helsinki, Finland). Sunflower oil was purchased from local supermarket without further purification. Milli-Q water was purified with a Millipore Synergy UV unit $(\mathrm{MQ} 18.2 \mathrm{M} \Omega \cdot \mathrm{cm})$ and used throughout the experiments.

Nanochitin Preparation. For simplicity, the chitin nanoparticles produced herein from mechanical methods are named as nanochitin $(\mathrm{NCh})$. The nanochitins were prepared following a reported protocol. ${ }^{39}$ Briefly, purified chitin was treated with 33 wt $\% \mathrm{NaOH}$ solution at $90{ }^{\circ} \mathrm{C}$ for $3.5 \mathrm{~h}$. The liquid-to-solid ratio used was $25 \mathrm{~mL} /$ g. This deacetylation step yielded partially deacetylated chitin (DEchitin), which was thoroughly washed with distilled water to reach neutral $\mathrm{pH}$ and further dried at room temperature. The degree of deacetylation (DD) of DE-chitin was $27.3 \%$, as determined by conductivity titration (Figure S1a). Before mechanical nanofibrillation, DE-chitin was redispersed in MQ water at a concentration of 0.2 wt \%, followed by $\mathrm{pH}$ adjustment (3.0) with acetic acid under vigorous stirring, to fully protonate the obtained amine groups. The obtained coarse suspension was homogenized to fine suspension by using a high-speed blender (T-25 Ultra-Turrax Digital Homogenizer, IKA, Germany) at room temperature. Two mechanical methods, ultrasonication and microfluidization, were used to disintegrate the DE-chitin into NCh.

Microfluidization of the fine suspension was carried out with a microfluidizer (M-110P, Microfluidics In., Newton, MA, U.S.A.) using a single pass at a pressure of 1500 bar. $^{40}$ The obtained nanofibrillated 
chitin was centrifuged at $10000 \mathrm{rpm}$ for $5 \mathrm{~min}$ to remove large particles, and the supernatant was collected as a dispersion with chitin nanofibers of the largest axial ratio or length, NCh-L. Ultrasonication was applied to the fine suspension using a titanium tip sonicator (Sonifier 450, Branson Ultrasonics Co., Danbury, CT, U.S.A.) for the given time at a power level set at $50 \%$ strength with alternating on-off cycles (5-2 s, respectively). The sonication time, 20 and $40 \mathrm{~min}$, was set to produce nanoparticles of medium and small axial ratio, NCh-M and NCh-S, respectively.

It should be noted that no other atoms besides $\mathrm{C}, \mathrm{N}$, and $\mathrm{O}$ were detected in the final NCh suspensions via SEM-EDX (Figure S1b). This points to the possibility that acetate (Ac) counterions were present in the aqueous suspension of the positively charged $\mathrm{NCh}$. This is explained to be the result of the acidification that was carried out with acetic acid, as was recently observed for chitin nanoparticles. $^{41}$

Nanochitin Morphology. The morphology of NCh was observed by transmission electron microscopy (TEM, JEM-2800, JEOL, Japan). A drop of diluted NCh suspension (0.005\%) was deposited on the electron microscope grid coated with carbon-reinforced formavar film, and negatively stained by uranyl acetate solution before drying at room temperature. Observation was conducted at an acceleration voltage of $120 \mathrm{kV}$. The length and width of NCh was measured via ImageJ software (imagej.nih.gov) by counting at least 100 nanoparticles (Figures S2 and S3). ${ }^{42}$

Surface and Interfacial Tension of NCh Suspensions. The surface and interfacial tension were measured with an optical tensiometer (Attension Theta, Biolin Scientific, Espoo, Finland) by injecting an air bubble in the NCh suspension via a hook needle. NCh suspensions at given concentrations were placed in a glass container and its transmissivity was checked prior to measurement. Briefly, an air bubble of $10 \mu \mathrm{L}$ was injected at the tip of hook needle immersed in the NCh suspension. After equilibrating the formed bubble for $30 \mathrm{~min}$ at room temperature, the surface tension value was calculated according to the bubble shape that was recorded during equilibrium. In addition, interfacial dilatational rheology (IDR) was applied by using the pulsating drop method (piezoelectric transducer, PD-200). Thus, the effect of any adsorbed nanoparticle was monitored under deformation. The total recording duration was $20 \mathrm{~s}$ for IDR with an oscillation frequency of $0.5 \mathrm{~Hz}$; the oscillation data between 4 to $16 \mathrm{~s}$ was chosen for building the isotherms. The interfacial tension was measured by the same method using sunflower oil. All experiments were carried out with freshly prepared samples and run in duplicate.

NCh-Stabilized Pickering Emulsions. NCh-stabilized Pickering emulsions were prepared using either sunflower oil or dodecane with the given NCh suspensions as stabilizer. The concentration of sunflower oil was varied from 10 to $50 \mathrm{wt} \%$ and $\mathrm{NCh}$ with different axial ratios, NCh-S, -M, and -L, were used. To dilute NCh suspension to given concentration, MQ water at $\mathrm{pH} 3.0$ (acetic acid) was added. Sunflower oil was loaded to NCh suspension in a plastic tube and the emulsion was formed by ultrasonication with a titanium tip sonicator with microtip being positioned close to the fluid surface. The power was set at $40 \%$ strength (determined by heat balance) and used alternating on-off cycles (3-2 s, respectively). The tube was immersed in ice-water mixture to avoid overheating. Unless stated otherwise, the concentration of $\mathrm{NCh}$ reported herein is based on the mass of the aqueous phase.

To visualize NCh and sunflower oil simultaneously, the oil phase was stained with Nile red before emulsion preparation. Nile red solution $(1 \mathrm{mg} / \mathrm{mL}$ in ethanol) was mixed with sunflower oil at a ratio of $1 / 25$, which was thoroughly stirred overnight. Similar preparation procedure was used to prepared the stained emulsions. The stained samples were stored at $4{ }^{\circ} \mathrm{C}$ before characterization. Optical photographs of emulsions were taken after storage at room temperature for $24 \mathrm{~h}$. The emulsion stability was monitored similarly after storage for 21 and 60 days. Some samples were kept for longer times to confirm longer term stability

Pickering Emulsion Characterization. Droplet Sizing. The mean droplet diameter and size distribution of Pickering emulsions were measured using a static light scattering instrument (Mastersizer
2000, Malvern Instruments, Malvern, U.K.). The emulsion was diluted with MQ water ( $\mathrm{pH}$ 3.0, acetic acid) prior to analysis to avoid multiple scattering effects. The refractive indices of sunflower oil and aqueous phase used in the calculation were 1.47 and 1.33, respectively. The mean droplet diameter of each sample was represented as the Sauter mean diameter $\left(D_{32}=\sum n_{i} d_{i}^{3} / \sum n_{i} d_{i}^{2}\right)$ that was calculated from the full droplet size distribution. All measurements were performed in triplicate after $24 \mathrm{~h}$ storage at room temperature.

Emulsion Morphology. The morphology of droplets after 60-day storage was examined using confocal laser scanning microscopy (CLSM) with a $63 \times$ oil immersion objective lens (Leica DMRXE, Leica, Germany). One hundred microliters of oil droplets (top layer) were stained with $10 \mu \mathrm{L}$ of Nile red solution prior to observation. After homogeneously mixing with a pipette and equilibrating for 10 min at room temperature, $6 \mu \mathrm{L}$ of the dyed sample was placed on a microscope slide and covered with a glass coverslip (Assistent, Sondheim, Germany). The coverslip was quickly fixed by nail polish to avoid evaporation. The excitation and emission spectrum for Nile red are 488 and $539 \mathrm{~nm}$, respectively.

The simultaneous observation of $\mathrm{NCh}$ and sunflower oil in emulsions was achieved with a Zeiss Axio Observer optical microscope (Zeiss, Germany) with a $100 \times$ oil immersion objective. Sunflower oil was stained by Nile red before emulsion preparation. The NCh was stained by Calcofluor white prior to observation, and the sample preparation procedure was similar as described for CLSM. The excitation and emission spectra for Calcofluor white stain were recorded at 365 and $435 \mathrm{~nm}$, respectively. Merged fluorescent images were processed by ImageJ. It should be noted that since the sample volume in the glass slide was larger than that needed to observe a single droplet layer, droplet overlapping often occurred, obscuring the interpretation (artifact images that appeared to occur from flocculation). Therefore, the droplet distribution was assessed with simultaneous experiments by CLSM.

The emulsion droplets prepared from NCh-L at low concentrations were visualized by optical microscopy (Leica DM 750, Leica, Germany) with a $10 \times$ objective lens. A drop of emulsion was dripped onto a microscope slide and covered with a glass coverslip.

Surface Morphology of Emulsion Droplets. To visualize the droplet surface, NCh-stabilized polystyrene (PS) beads that were produced from polymerization of styrene ( $\mathrm{St}$ )-in-water emulsions were prepared. Briefly, suspensions containing 0.3 wt \% NCh-S, $-\mathrm{M}$, or $-\mathrm{L}$ were used as aqueous phase. St was mixed with an oil-soluble initiator, azobis(isobutyronitrile) (AIBN), to obtain a St/AIBN ratio of $100 / 1(\mathrm{w} / \mathrm{w})$. A total of $1.0 \mathrm{~g}$ of this mixture was added to $9.0 \mathrm{~g}$ of $\mathrm{NCh}$ suspension. The emulsions were prepared as standard procedure. Before polymerization at $65{ }^{\circ} \mathrm{C}$, emulsions were degassed with nitrogen gas for $10 \mathrm{~min}$. The reaction was performed for $24 \mathrm{~h}$ without stirring. The PS beads obtained were observed by scanning electron microscopy (SEM, Zeiss Sigma VP, German) operated under vacuum and at an accelerated voltage of $2 \mathrm{kV}$. The surface of dried PS beads was sputter-coated with platinum/palladium.

Rheological Behavior. The apparent viscosity of emulsions was measured with a rheometer (MCR 302, Anton Paar, Germany) operating with parallel plates (PP25) using a $0.5 \mathrm{~mm}$ gap. All emulsions were presheared at $10 \mathrm{~s}^{-1}$. The shear viscosity was monitored at increased shear rates (from 0.01 to $100 \mathrm{~s}^{-1}$ ). The flow curves were fitted to the Herschel-Bulkley model, ${ }^{43}$ which is commonly applied to emulsified systems:

$$
\tau=\tau_{0}+k \gamma^{n}
$$

where $\tau$ and $\tau_{0}$ are the shear and yield stress, respectively (both in $\mathrm{Pa}$ ), $k$ is the consistency $\left(\mathrm{Pa} \cdot \mathrm{s}^{n}\right)$ and $n$ is the flow indices, respectively.

For dynamic viscoelastic measurements, the linear viscoelastic range was determined using the same rheometer and geometry described above via a strain sweep $(0.01-100 \%)$ at a fixed $10 \mathrm{rad} / \mathrm{s}$ frequency. After this, a dynamic frequency sweep was conducted by applying a constant strain of $1.0 \%$, which was within the linear region, and over a frequency range between 0.1 and $100 \mathrm{rad} / \mathrm{s}$. The dynamic 


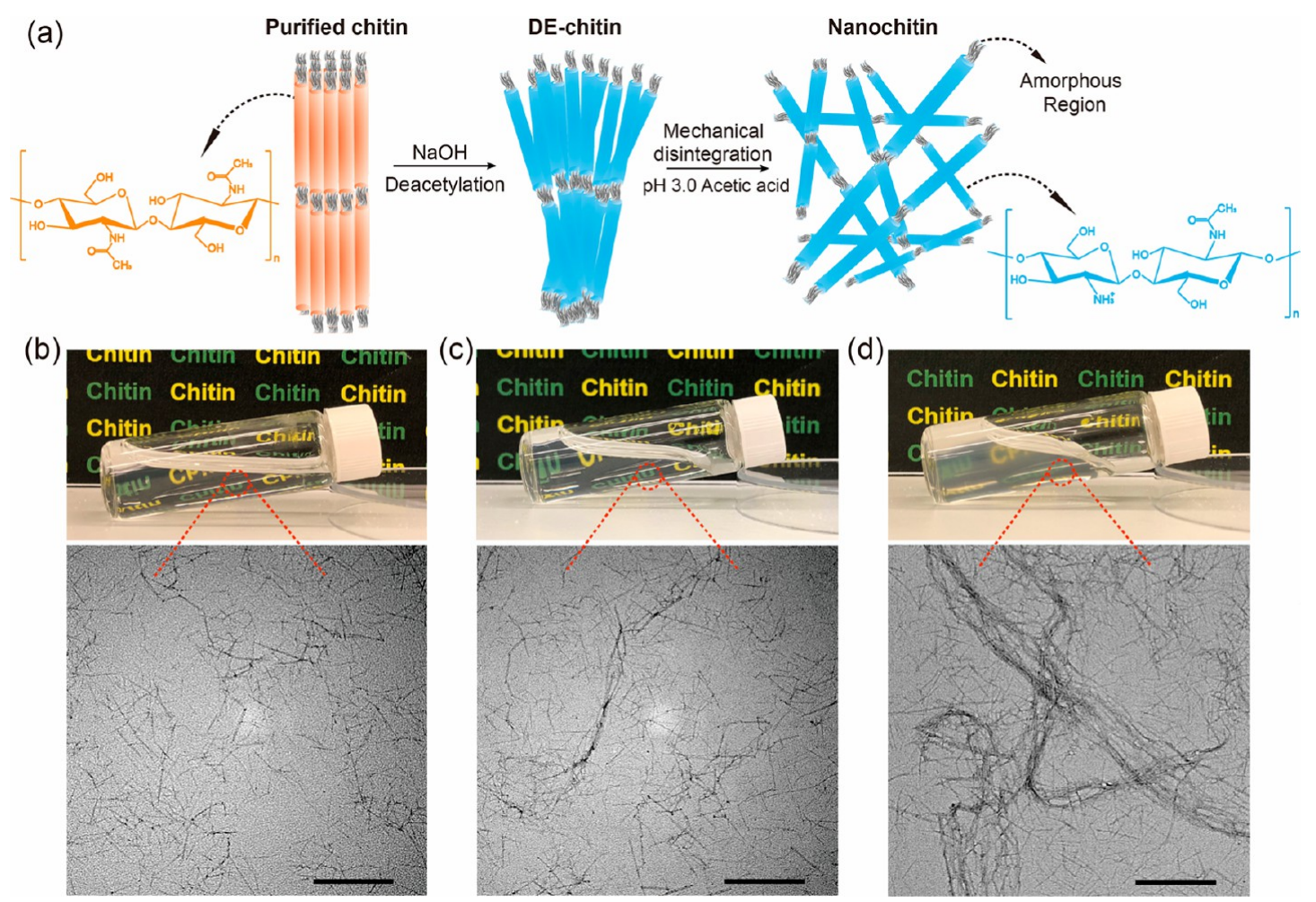

Figure 1. (a) Illustration (not to scale) of nanochitin ( $\mathrm{NCh}$ ) preparation via mechanical disintegration (ultrasonication or microfluidization) of $\mathrm{NaOH}$-mediated deacetylated chitin under acidic condition. Visual appearance (top) and TEM micrograph (bottom) of the as-prepared NCh suspension ( $\mathrm{pH}$ 3.0, acetic acid) with different axial ratio: (b) short (NCh-S), (c) Medium (NCh-M) and (d) long (NCh-L) NCh. The concentration of $\mathrm{NCh}$ for visual characterization was kept at $0.3 \mathrm{wt} \%$. The scale bar is $500 \mathrm{~nm}$.

mechanical profiles were obtained by recording the storage $\left(G^{\prime}\right)$ and the loss $\left(G^{\prime \prime}\right)$ moduli as a function of frequency. All measurements were performed at $25{ }^{\circ} \mathrm{C}$.

\section{RESULTS AND DISCUSSION}

Nanochitin Properties. Morphology. Figure 1a schematically shows the preparation of nanochitin $(\mathrm{NCh})$, which displays the use of deacetylated chitin (DE-chitin) to generate $\mathrm{NCh}$ in acidic condition and after mechanical disintegration. This process simultaneously achieves partial shifting of surface $\mathrm{N}$-acetyl groups to protonated amino groups, resulting in positively charged NCh. Particularly, the deacetylation of Nacetyl groups is a nonselective process, ${ }^{32}$ thereby generating a random distribution of amino groups. It is worth noting that the nonordered domains of chitin chains in $\mathrm{NCh}$ are likely to be preserved because no hydrolysis occurs. Nanochitins with different axial ratios were isolated from the same batch of DEchitin. The axial ratio was defined on a relative basis as small $(-\mathrm{S})$, medium (-M), and large (-L) as obtained either by ultrasonication (NCh-M and $-\mathrm{S}$ ) or microfluidization (NCh-L) (Figure $1 \mathrm{~b}-\mathrm{d}$ ). The positive $\zeta$-potential produced from the cationization of NCh by the different methods was remarkably high and similar, $105 \pm 4.5 \mathrm{mV}$, indicating that the degree of deacetylation $(\sim 27 \%)$ was not altered under mechanical processing. Accordingly, it can be postulated that similar surface chemical properties exist for different samples, such as the residual $\mathrm{N}$-acetyl groups of nanochitins $(-\mathrm{S},-\mathrm{M},-\mathrm{L})$.

TEM micrographs (Figure $1 \mathrm{~b}-\mathrm{d}$, bottom, and Figure S2) of the NCh samples indicated well dispersed nanoparticles (fibrils) in aqueous media with no signs of aggregation even for the longest NCh (NCh-L). This is attributed to the strong electrostatic repulsion that originated from the highly cationic surface charge. Figure S3a,b for NCh-S indicate the smallest nanoparticle size given the more severe ultrasonication used. Interestingly, the width of $\mathrm{NCh}-\mathrm{S}$ and $-\mathrm{M}$ was similar (approximately $10 \mathrm{~nm}$ ) (Figures S2 and S3b,d), which was close to that of individual chitin nanofibrils, ${ }^{44}$ implying that lateral disassembling of chitin was dominant due to the strong repulsion between nanoparticles upon cationization. Curled, loosely entangled as well as straight nanofibrils were observed in the NCh-L sample with TEM widths ranging from 15 to 100 $\mathrm{nm}$ (wider compared to those of NCh-S and -M) and with lengths in micrometer range or larger (Figure 1d). The morphologies observed were the result of the microfluidization disintegration mechanism used to obtain NCh-L: ${ }^{45}$ the collision of separated streams in the unit produce high shear, depending on the pressure applied. ${ }^{46}$ Under the conditions of low energy shearing (minimum pressure and single pass), the cationized chitin nanoparticles underwent relative low severity deconstruction conditions as far as length reduction and lateral bundle disassembly. However, we note that chitin nanorods of smaller sizes were also present in NCh-L suspension, indicating their high size polydispersity, as it is typical from random processes during microfluidization (for instance, as observed in the case of CNF). ${ }^{47}$ The results point to the fact that NCh can be easily obtained with varying average axial ratio and under conditions that are much less severe than those that apply to other biomass-derived materials. According to Figure S3, the length and width of NCh-S were $166 \pm 25$ and $11 \pm 2 \mathrm{~nm}$, respectively. For $\mathrm{NCh}-\mathrm{M}$, the respective values were $261 \pm 35$ and $11 \pm 3 \mathrm{~nm}$. Thus, the corresponding axial ratio (length/ width, $L / w)$ was $\sim 15$ and $\sim 25$ for NCh-S and NCh-M, respectively. Given the complex microstructure of NCh-L, it was difficult to accurately measure the length and width of 

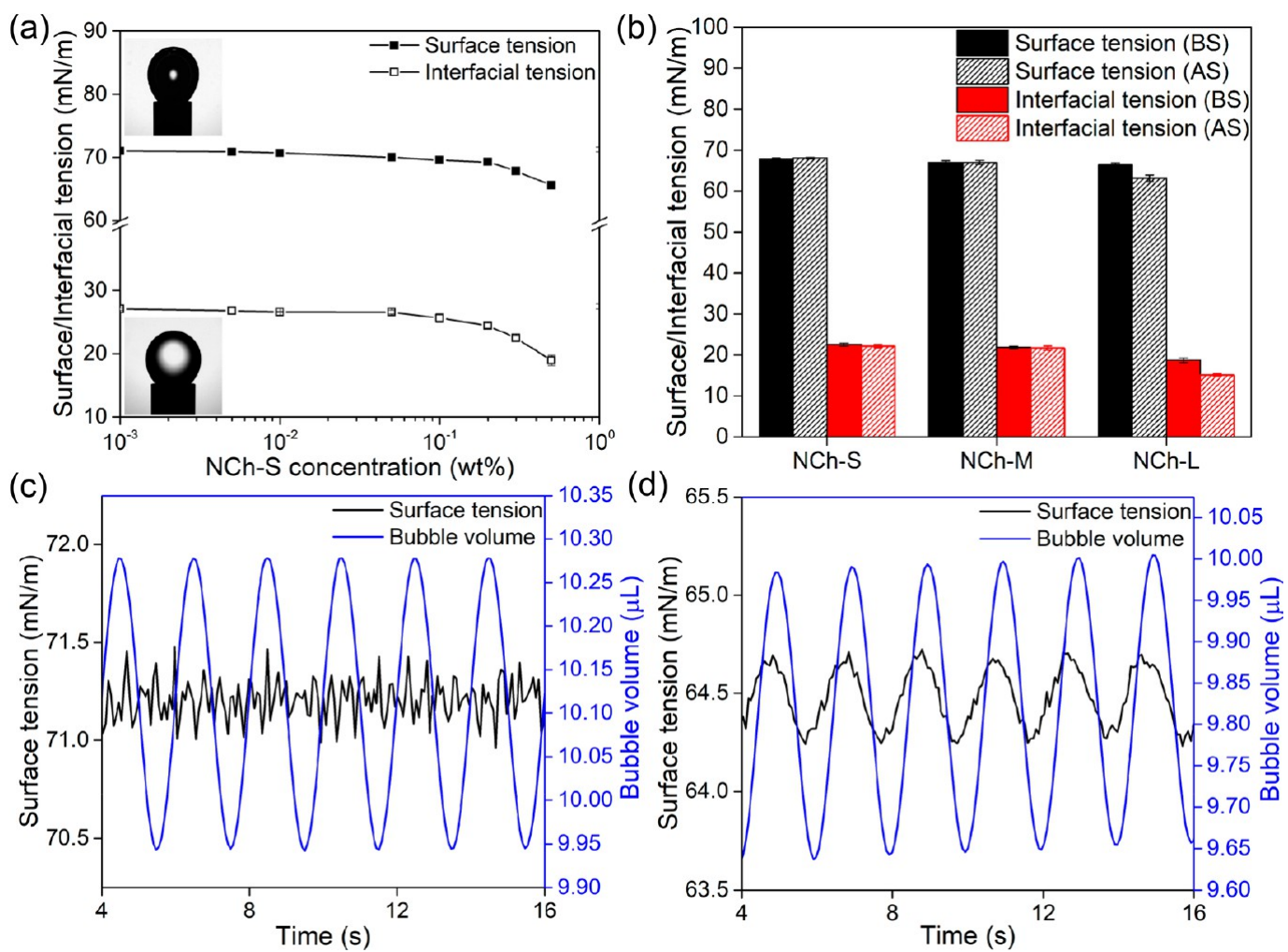

Figure 2. Surface (air-water) and interfacial (sunflower oil-water) tension of (a) NCh-S suspension at varying concentrations and (b) NCh suspension with different axial ratio before and after additional $90 \mathrm{~s}$ sonication at $0.3 \mathrm{wt} \%$. The BS and AS abbreviations in (b) refer to before- and after-sonication, respectively. (c) 0.001 and (d) $0.5 \mathrm{wt} \% \mathrm{NCh}-\mathrm{S}$ for interfacial dilatational rheology measurement at air-water interfaces with oscillation frequency at $0.5 \mathrm{~Hz}$. All the measurements were conducted at room temperature.

individual NCh-L nanoparticles. However, on the basis of individual NCh-L and bundles that could be identified from TEM micrographs, an average width and length of $39 \pm 28$ and $2270 \pm 1600 \mathrm{~nm}$ were obtained (based on 100 measurements from ImageJ). Thus, it can be estimated that the axial ratio for NCh-L was $L / w \sim \geq 60$, much larger than those of NCh-S and -M. Admittedly, the actual axial ratio of NCh-L in suspension might significantly vary due to the existence of irregular bundles. The NCh suspensions observed at the same solid concentration displayed increased light scattering (increased turbidity), owing to the increased axial ratio, in the order NCh$\mathrm{L}>\mathrm{NCh}-\mathrm{M}>\mathrm{NCh}-\mathrm{S}$ (Figure $\mathrm{lb}-\mathrm{d}$, top).

Surface and Interfacial Activity. The surface (air-water) and interfacial (sunflower oil-water) tension of NCh samples were determined with an optical tensiometer with a hook needle configuration (Figure 2). In this method, an air bubble is generated inside an aqueous $\mathrm{NCh}$ suspension to minimize the external effects that otherwise apply when a drop of the suspension is generated in air. Figure 2 a clearly indicates the effect of NCh-S in reducing the surface tension at concentrations above $0.3 \mathrm{wt} \%$. Since the optical assessment may be subjected to effects that are not fully controlled during recording of the images, the surface activity of NCh-S was also measured by interfacial dilatational rheology (IDR). For IDR (Figure 2c,d), the blue (right axis) and black (left axis) profiles show the variation of the volume and surface tension of the pulsating air bubble during the measurement, respectively. Figure $2 \mathrm{c}$ indicates that the surface tension for $0.001 \mathrm{wt} \%$ $\mathrm{NCh}-\mathrm{S}$ was fairly constant during oscillation $(\sim 71.0 \mathrm{mN} / \mathrm{m})$, for example, no chitin nanoparticles were adsorbed at the airwater interface (Figure 2a). However, at higher concentrations
(0.5 wt \% NCh-S), the surface tension followed the oscillation profiles corresponding to the bubble volume (Figure 2d). This indicates that NCh-S was effectively adsorbed at the air-water interface and the adsorbed layer was influenced by the deformation of the air bubble, demonstrating the ability of NCh-S to decrease surface tension (Figure 2a). The IDR results confirmed a reduction of surface tension at the given NCh-S concentration obtained from the optical tensiometer that used the hook needle. Taken together and compared to other biomass-derived nanoparticles, such as cellulose nanocrystals and nanofibers, the surface tension data shows a distinctive behavior for NCh-S.

The same sample, NCh-S, lowered the interfacial tension at very lower concentrations $(>0.05$ wt $\%$ ) (Figure $2 \mathrm{a}$ ), which can be attributed to the wettability that was originated from the hydrophobic-hydrophilic balance endowed by the surface $\mathrm{N}$ acetyl and amino groups, respectively. We note that the nature of sunflower oil and its partially oxidized components, may also contribute to this effect. ${ }^{48}$ As shown in Figure $2 b, \mathrm{NCh}-\mathrm{M}$ and $-\mathrm{L}$ also reduced the surface tension at $0.3 \mathrm{wt} \%$, showing similar surface activity to that of NCh-S. Compared to NCh-S and -M, $\mathrm{NCh}-\mathrm{L}$ was more efficient in lowering the interfacial tension, possibly resulting from the effects of its polydispersity. The ability to reduce interfacial tension within the tested concentrations indicate the potential of NCh to emulsify oil phases. In summary, NCh with different axial ratio can be controllably produced from mechanical disintegration, enabling a reproducible process toward fine $\mathrm{NCh}$ particles for uses in Pickering stabilization, as will be explored next.

Nanochitin-Based Pickering Emulsions. Formation and Stabilization of Pickering Emulsions. Pickering emul- 
(a)
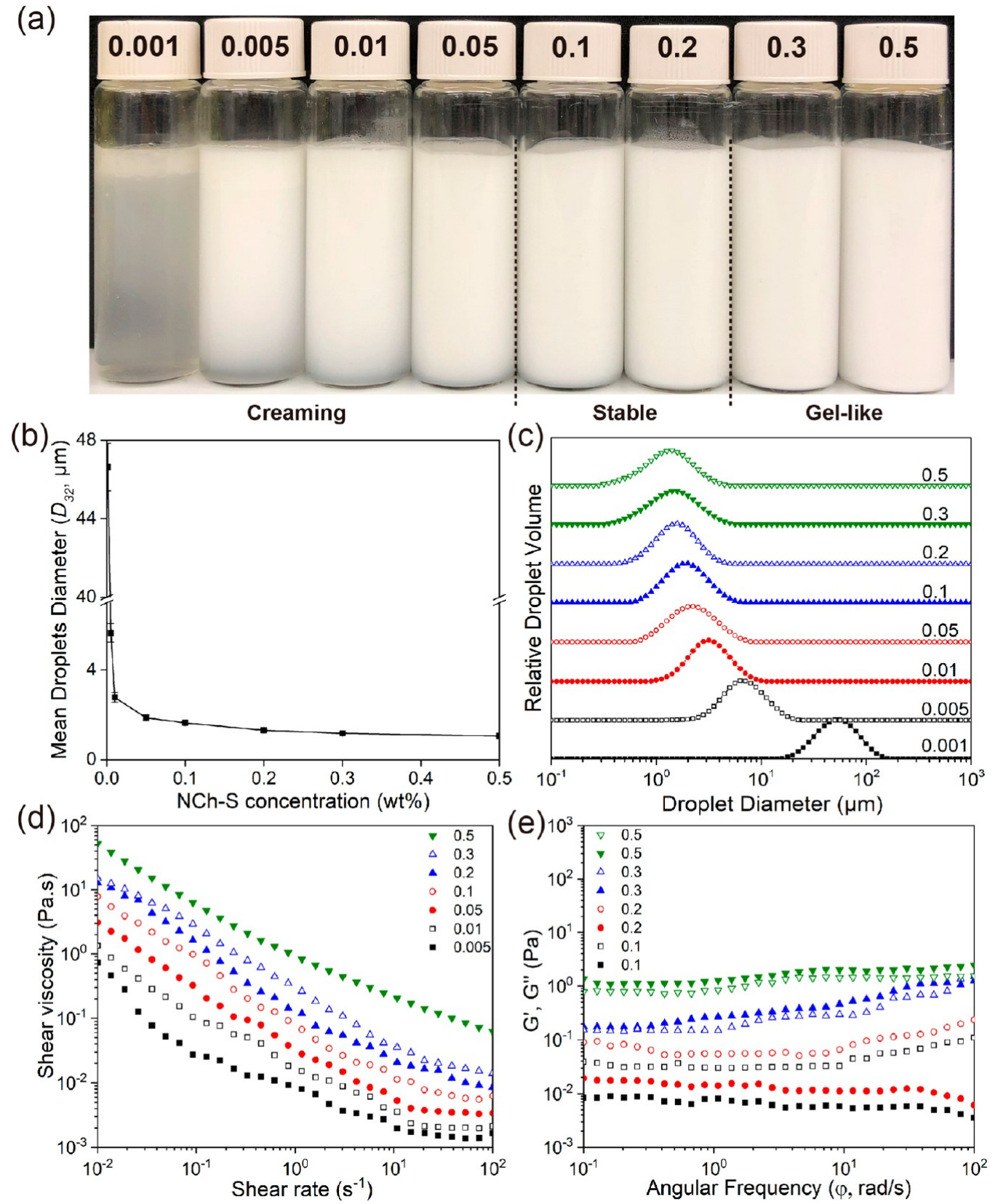

Figure 3. (a) Visual appearance, (b) mean droplet diameter $\left(D_{32}\right)$ and (c) diameter distribution of Pickering emulsions (10 wt \% oil) stabilized by NCh-S at concentrations of $0.001,0.005,0.01,0.05,0.1,0.2,0.3$, and 0.5 wt \% (see labels in the respective vial in (a)). In (a), black dotted lines were added to indicate three distinctive emulsion regimes. All samples were stored at room temperature for $24 \mathrm{~h}$ before characterization. (d) Flow profiles and (e) modulus for NCh-S-stabilized Pickering emulsions containing varied NCh-S concentrations (wt \%). For better visualization, open and filled symbols were used in (d) with the storage $\left(G^{\prime}\right)$ and loss $\left(G^{\prime \prime}\right)$ moduli shown with filled and open symbols, respectively. The emulsion containing 0.001 wt \% NCh-S was phase-separated during the rheological test.

sions prepared from NCh-S suspension and sunflower oil (10 wt \%) were prepared using various NCh-S concentrations (Figure 3), and their microstructure was observed by fluorescent microscopy (Figure 4). As shown in Figure 3a, Pickering emulsion stabilized at ultralow NCh-S concentration ( $0.001 \mathrm{wt} \%$, or $0.0005 \mathrm{wt} \%$ of the total) was stable against oiling off upon storage for $24 \mathrm{~h}$. Slight creaming with formation of a bottom aqueous serum was observed at low NCh-S concentrations ( 0.005 to 0.05 wt \%), which can be attributed to the relatively large droplets that were formed (Figure $3 \mathrm{~b}$ ) and less viscous aqueous phase (Figure 3d). By increasing NCh-S loading (>0.1 wt \%), emulsions turned to be stable without any creaming. This can be ascribed to the change in rheological behavior of the aqueous phase (Figure $3 \mathrm{e}$ ). The Herschel-Bulkley model fitted the shear stress-shear rate profiles of the emulsions at different NCh-S concentrations (see yield stress, consistency, and flow indices in Table S1) with a determination coefficient $\left(R^{2}\right)>0.98$ for all samples. The flow index $(n)$ varied from 0.04 to 0.57 , indicating that the emulsions were pseudoplastic or displayed a shear-thinning behavior.

Oil droplets were well dispersed in aqueous phase after $24 \mathrm{~h}$ storage, Figure 4, indicating good stabilizing ability of NCh-S. Furthermore, according to the merged images (see blue 


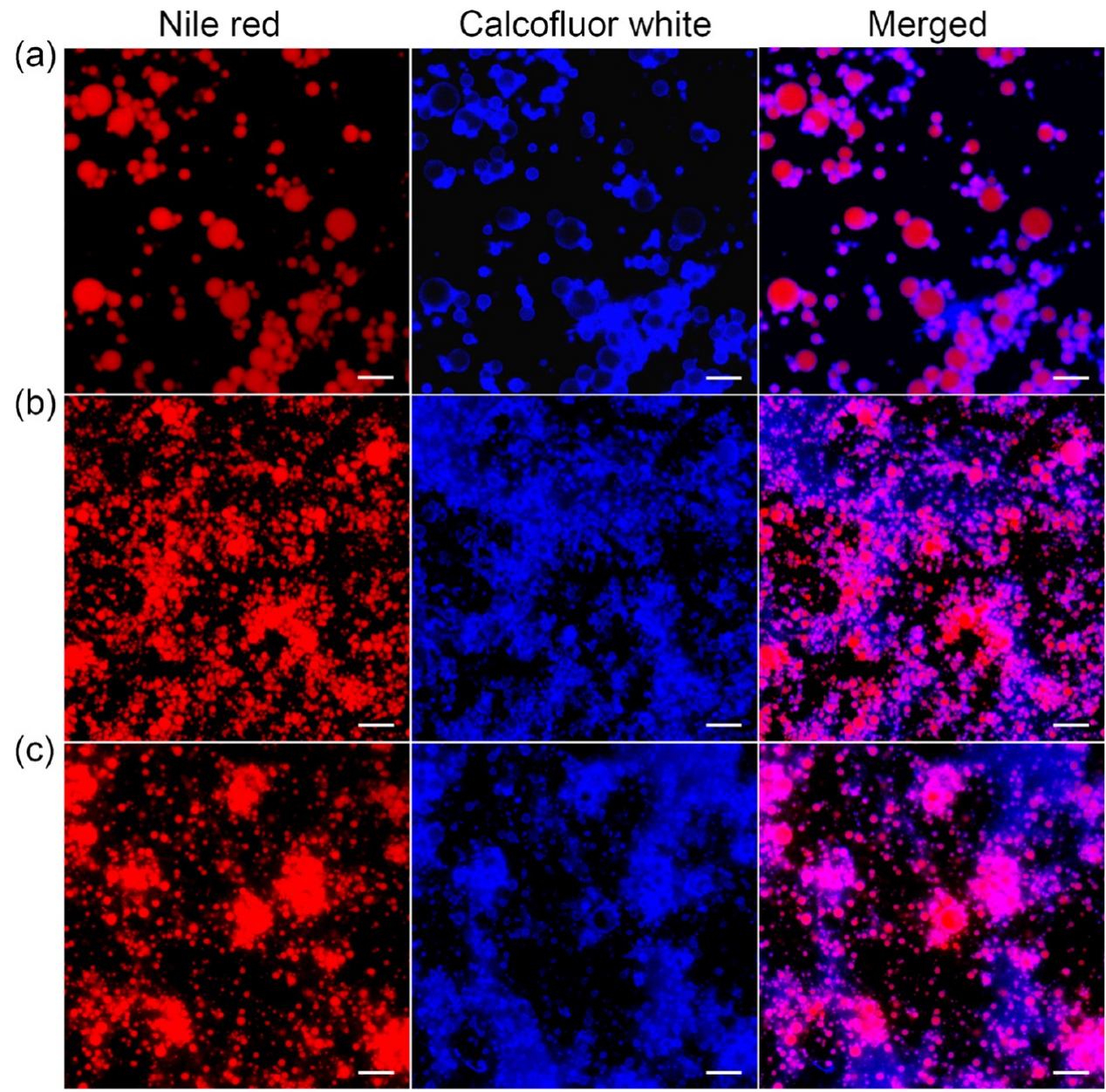

Figure 4. Fluorescent micrographs of Pickering emulsions (10 wt \% sunflower oil) stabilized with NCh-S at concentration of (a) 0.005, (b) 0.2, and (c) $0.5 \mathrm{wt} \%$. The left, middle, and right rows correspond to the stained oil phase, dyed NCh-S, and the merged images, respectively. The scale bar is $10 \mu \mathrm{m}$. All samples were stored at room temperature for $24 \mathrm{~h}$ before observation.

contour around oil droplets, Figure 4), NCh-S was located at the oil-water interface, regardless of concentrations, which can be taken as a direct proof of the role of NCh in Pickering emulsion stabilization. The mean droplet diameter $\left(D_{32}\right)$ of Pickering emulsions sharply decreased from $\sim 47$ to $\sim 5 \mu \mathrm{m}$ upon increasing NCh-S concentration from 0.001 to $0.005 \mathrm{wt}$ $\%$ (based on mass of the aqueous phase), Figure $3 \mathrm{~b}$. By further increasing NCh-S addition over 0.05 wt \%, the droplet size reached a plateau at approximately $1.5 \mu \mathrm{m}$ (and down to 1.1 $\mu \mathrm{m}$ at 0.5 wt \% NCh-S). The size distribution for all NCh-S concentrations was unimodal with approximately the same peak width, indicating similar size polydispersity, Figure 3c. These results can be related to the surface coverage of NCh-S on the oil droplets. The surface coverage ratio of NCh-S for the different formulations was calculated following approximated geometrical considerations (Table S2). ${ }^{14}$ At low NCh-S addition $(\leq 0.05 \mathrm{wt} \%)$, the surface coverage for the stable droplets was relatively low, indicating that the expected small emulsion droplets that were initially generated during ultrasonication, underwent coalescence and size growth. ${ }^{49}$ However, once coalescence ceased, that is, upon reaching a sufficient coverage, the droplets became stabilized by the interfacial barrier introduced by adsorbed NCh-S. At 0.5 wt \% NCh-S loading, the calculated coverage ratio was $>100 \%$, which indicated the presence of free NCh-S in the aqueous phase, as confirmed in Figures $3 c$ and $S 4 b$. Indeed, the theoretical concentration required to achieve full coverage of the formed oil droplets (with $1.1 \mu \mathrm{m}$ average diameter) is approximately 0.39 wt \%.

A reason for the droplet stability observed even at low surface coverage is the ability of NCh-S to organize and to form connected networks at the interfaces (see Figure 7a), thereby reducing the demand for otherwise a high surface coverage needed to prevent oil fusion. ${ }^{38}$ The emulsion droplet size was fairly similar at NCh-S concentrations of $0.1 \mathrm{wt} \%$ or larger. At this concentration (0.1\%), the NCh-S surface coverage is $\sim 40 \%$ (Table S2), effectively restricting coalescence of newly generated oil droplets (and thus keeping the droplet size unchanged throughout preparation). NCh-S is highly efficient in forming robust nanostructures and interfacial barriers that stabilize the dispersed phase in the emulsion. The $40 \%$ coverage calculated for stable emulsions at $0.1 \mathrm{wt} \% \mathrm{NCh}-$ $\mathrm{S}$ concentration is comparatively lower than the $84 \%$ required in the case of other biobased, rodlike Pickering stabilizers, such as cellulose nanocrystals, CNC, with similar dimensions. ${ }^{38}$ Thus, in the case of $\mathrm{NCh}$, much less material is required to adsorb at the interface, indicating a better emulsifying ability, at least for the present set of conditions.

The $\zeta$-potential of oil droplets in emulsions stabilized with different NCh-S concentrations was quite similar, $80 \pm 7 \mathrm{mV}$, providing large electrostatic interdroplet repulsion. Together with the ability of NCh-S to lower the interfacial tension, the 
(a)

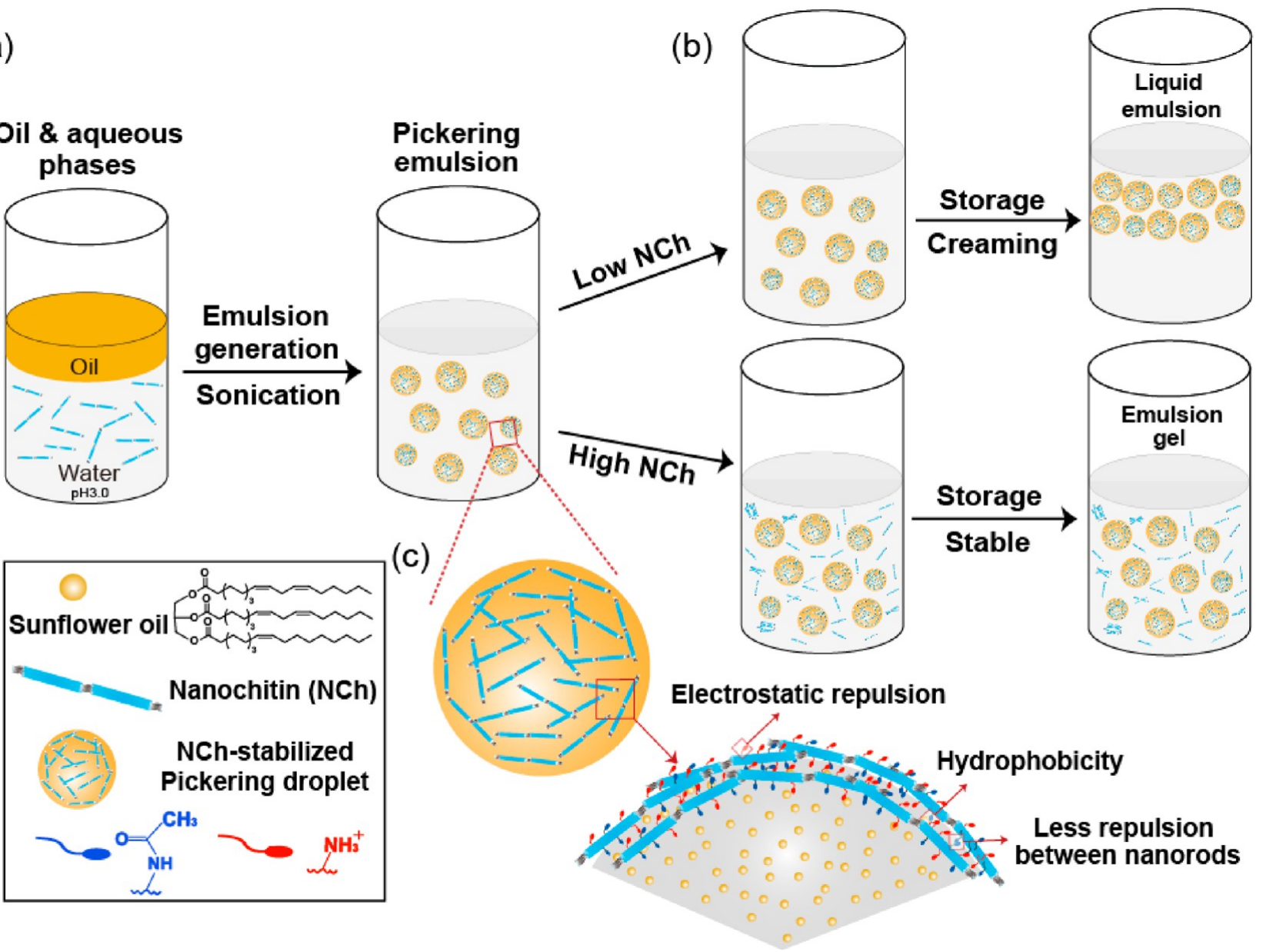

Figure 5. Schematic illustration (not to scale) of (a) Pickering emulsion formation via nanochitin adsorption at sunflower oil-water interface and (b) two different loading levels of nanochitin and the corresponding storage stability. (c) Possible stabilization behavior of NCh at interfaces. Random distribution of hydrophobic $\mathrm{N}$-acetyl and protonated amino groups increases interfacial wettability and reduces the interparticle repulsion, promoting NCh interfacial adsorption.

two effects contribute to the formation of small droplets. ${ }^{14}$ It should be noted that no electrolyte was added in this system to screen the surface charge of NCh-S before emulsion preparation, which is otherwise applied for highly charged stabilizers, for example, to promote interfacial assembly (the case of CNC). ${ }^{38}$ As discussed previously, NCh simultaneously bears hydrophobic $\mathrm{N}$-acetyl and hydrophilic amino groups, which are randomly distributed on their surfaces. Accordingly, it can be concluded that the coexistence of such groups on $\mathrm{NCh}-\mathrm{S}$ provides a right balance of interfacial wettability and adsorption at the oil-water interface, Figure 5c. The random distribution of charged and uncharged groups on the NCh surface are hypothesized to undergo charge matching at close range distances, limiting the effect of the measured net electrostatic charge within the tightly covering NCh-S adsorbed at the interface. In sum, the formation and stabilization of fine droplets by adsorbing NCh-S, which prevents oiling off, results from a synergistic combination of high-efficient structuring of the chitin nanoparticles at the oilwater interface, a significant electrostatic repulsion, and the development of a strong interfacial steric barrier. In closing, and most remarkably, comparing the droplet size with those obtained from CNC-stabilized Pickering emulsions, ${ }^{49}$ an extremely low NCh concentration (0.001 wt \%) is sufficient to achieve stability against oiling off. Furthermore, the droplet size was much smaller for NCh-S at similar concentrations. These results clearly demonstrate the high efficiency, tunability, and feasibility of NCh as a biobased Pickering stabilizer.

To confirm the underlying stabilization mechanism, rheological measurements were performed with the emulsions (Figure 3d,e), which may explain their stability against creaming, even if oiling off is avoided. The flow curves of emulsions containing $10 \mathrm{wt} \%$ sunflower oil at varying NCh-S loadings showed a strong shear thinning (Table S1) with the apparent viscosity increasing with NCh-S concentration (Figure 3d). This is simply explained by the increased viscosity of the continuous phase (Figure S5a). Furthermore, besides the adsorbed NCh-S, free NCh-S was evident in the aqueous phase at high NCh-S loadings (merged images in Figure $4 \mathrm{c}$ and $\mathrm{S} 4 \mathrm{~b}$ ), which increased the viscosity of the continuous phase and thus that of the emulsions.

At NCh-S concentrations $\leq 0.2$ wt $\%$, the emulsion showed $G^{\prime}<G^{\prime \prime}$, Figure 3e, indicating a liquid-like behavior, which might be ascribed to the weak interparticle interaction since no free NCh was dispersed in the aqueous phase (Figure 4a). At higher NCh-S concentrations (>0.2 wt \%), $G^{\prime} \sim G^{\prime \prime}$, suggesting the onset of the formation of an emulsion gel. This effect may be ascribed to the increased interaction propensity between droplets since more chitin nanoparticles 

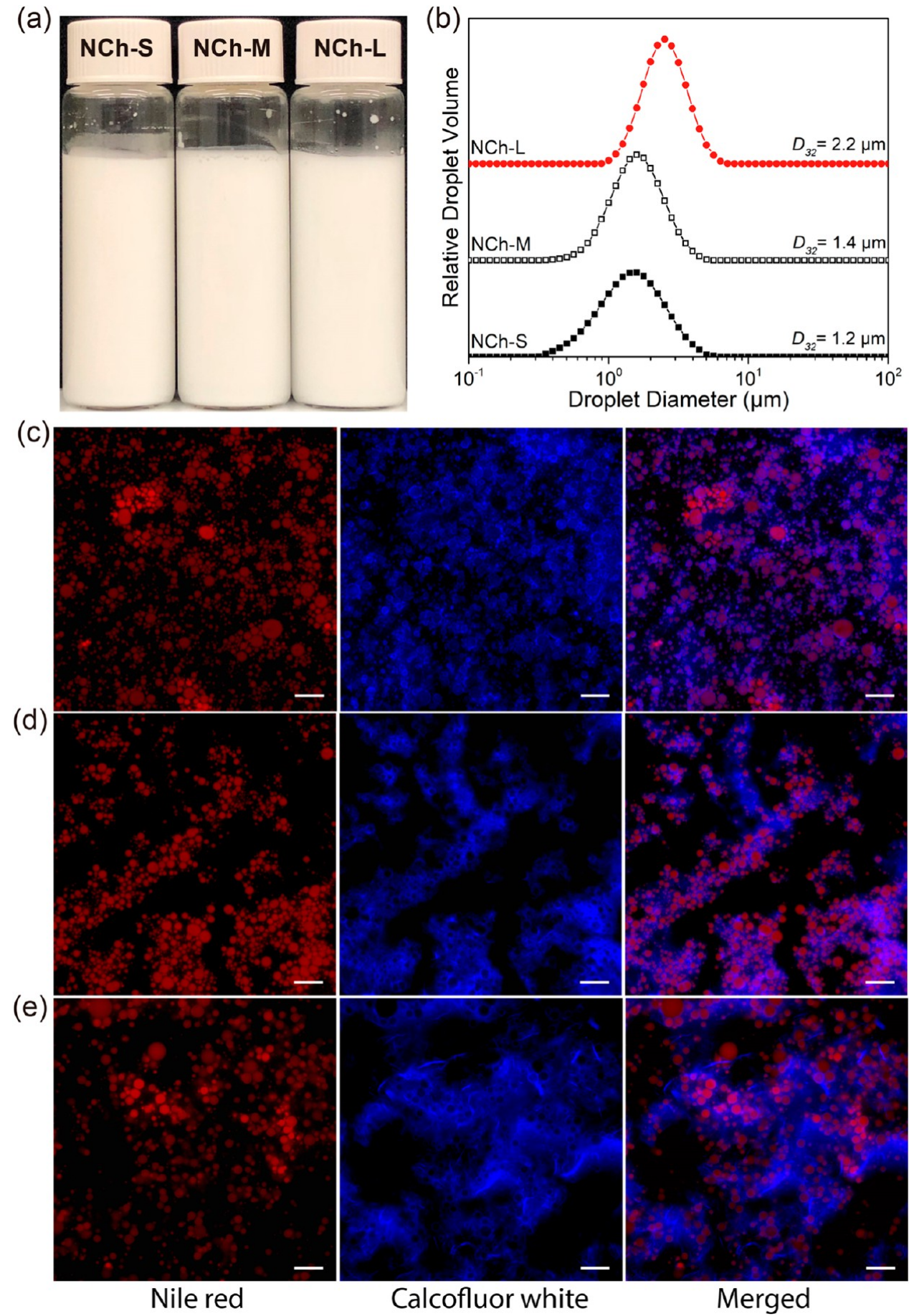

Figure 6. (a) Visual appearance and (b) size distribution of Pickering emulsions (10 wt \% oil) stabilized with NCh of different axial aspect: NCh-S, NCh-M, and NCh-L (as noted). The NCh concentration was $0.3 \mathrm{wt} \%$. Mean droplet diameter $\left(D_{32}\right)$ was indicated in (b). The fluorescent micrographs of emulsions stabilized with (c) NCh-S, (d) NCh-M, and (e) NCh-L. The left, middle and right rows correspond to the stained oil phase, dyed NCh, and merged images, respectively. The scale bar is $10 \mu \mathrm{m}$. All samples were stored at room temperature for $24 \mathrm{~h}$ before characterization.

were dispersed in the continuous phase (especially observed from the merged image in Figure S4b). ${ }^{26}$ As shown in Figure $\mathrm{S} 4 \mathrm{~b}$, the entanglement of longer nanoparticles in NCh-S at high concentration possibly triggers the formation of an emulsion gel. According to the results, two different regimes can be distinguished for NCh-S-stabilized Pickering emulsions: liquid-like emulsions ( $\leq 0.2 \mathrm{wt} \% \mathrm{NCh}-\mathrm{S})$ and emulsion gels (>0.2 wt \% NCh-S) (Figure $5 \mathrm{~b}$ ). The results explain that at low NCh-S concentration, a weaker aqueous phase resistance existed to otherwise limit the upward movement of oil droplets during creaming. At high NCh-S loadings, the formed emulsion gel kinetically traps the oil droplets and restricts their mobility, leading to homogeneous emulsions.

One question that remains is that at intermediate NCh-S concentrations ( 0.1 and $0.2 \mathrm{wt} \%)$, although a liquid emulsion was formed (Figure 3e), no creaming was observed after $24 \mathrm{~h}$ 

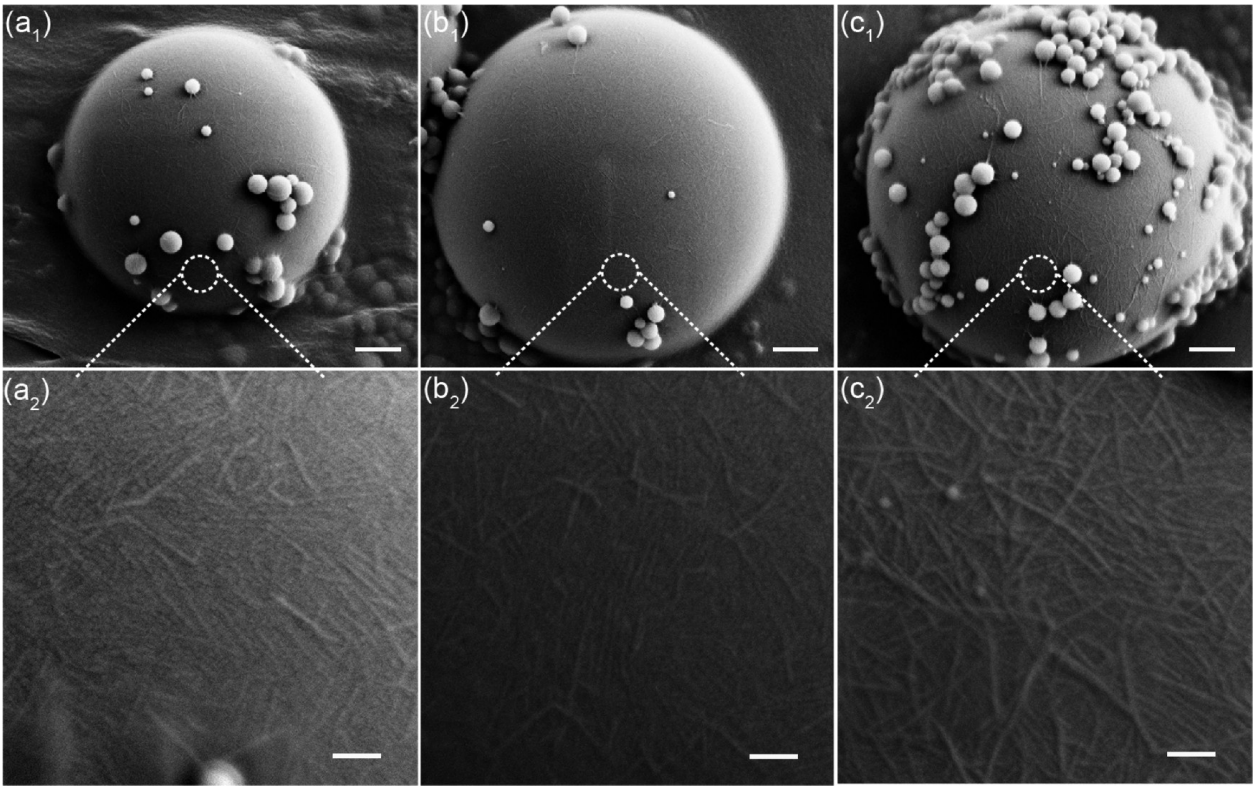

Figure 7. SEM images of polymerized styrene-in-water emulsions stabilized by NCh-S $\left(a, a_{1}\right)$, NCh-M (b, $\left.b_{1}\right)$, and NCh-L (c, $\left.c_{1}\right)$ at a concentration of $0.3 \mathrm{wt} \%$. The scale bar in $\mathrm{a}_{1}, \mathrm{~b}_{1}$, and $\mathrm{c}_{1}$ is $500 \mathrm{~nm}$, and that in $\mathrm{a}_{2}, \mathrm{~b}_{2}$, and $\mathrm{c}_{2}$ is $100 \mathrm{~nm}$.

storage (Figure 3a). On the basis of the above results and our previous work with $\mathrm{CNF}^{50}$ we hypothesize that emulsions stabilized at these concentrations resulted from the viscoelastic nature of the aqueous phase (thickening and microstructuring). Indeed, although emulsions are liquid-like, the viscous and viscoelastic properties of the continuous phase is more significant than those of lower NCh-S concentrations (Figure $3 \mathrm{~d}, \mathrm{e})$. Because the viscosity and elasticity of the aqueous phase surrounding oil droplets increased, the movement of droplets was prevented, at least temporarily: creaming was observed for the samples stabilized with NCh-S at 0.1 and 0.2 wt \% after storage for more than one month (see Figure S10, to be discussed later). Therefore, the change of the environment of the continuous phase induced by NCh-S and surrounding the oil droplets allows for its viscoelastic properties to kinetically restrict the mobility of droplets, which enables a temporary stable emulsion.

Pickering Emulsions at High Internal Phase Fraction. Figure S6 shows the properties of NCh-S-stabilized Pickering emulsions at given oil phase fraction or water-to-oil ratio (WOR). As shown in Figure S6a, emulsions prepared with identical NCh-S concentration ( 0.5 wt \%) but different oil fractions were stable against creaming and oiling off after storage for $24 \mathrm{~h}$. The droplet diameter shown in Figure S6b indicates that the oil droplet size gradually increased with increasing oil content, reaching $4.6 \mu \mathrm{m}$ at an oil fraction of 0.5 . Furthermore, the size distribution of emulsion droplets was unimodal at all oil fractions, for example, the droplet size was homogeneous in all samples. By increasing the oil phase fraction at given NCh-S concentration, the relative nanoparticle coverage was reduced, thereby increasing the possibility for droplet coalescence during ultrasonication, which resulted in larger droplets. Figure S7a,b shows the rheological behavior of emulsions at different oil fractions, revealing the shear thinning nature of all emulsions $(n>1$, Table S1) and $G^{\prime}>G^{\prime \prime}$, indicating the formation of emulsion gels. This resulted from the relatively high NCh-S loading, as well as the reduced free volume and increased interdroplet interaction when the volume of dispersed phase was increased (higher oil fraction), producing larger droplet number density. They are also the reasons to explain the high emulsion stability during storage. In summary, NCh-S is an efficient stabilizer of Pickering emulsions even at high oil fraction, which is desirable in practical applications.

Effect of NCh Axial Ratio. Emulsions prepared from NCh (0.3 wt \%) with different axial ratios were investigated (Figure 6). All produced emulsions were stable during storage for $24 \mathrm{~h}$, Figure 6a. The average size of oil droplets stabilized by NCh-S and $-\mathrm{M}$ was similar, Figure $6 \mathrm{~b}$, but it increased to $2.2 \mu \mathrm{m}$ for those stabilized by NCh-L. This is likely due the relative lower $\mathrm{NCh}-\mathrm{L}$ number density in the precursor aqueous medium at the given mass concentration (less NCh-L nanoparticles are available to stabilize oil phase and thus lower surface coverage is determined compared to that of $\mathrm{NCh}-\mathrm{S}$ ), leading to coalescence of newly generated droplets during preparation. This is confirmed by experiments carried out with styrene (St)in-water emulsions stabilized with the NCh followed by polymerization into solid beads to facilitate imaging of $\mathrm{NCh}$ nanofibrils at the interface, Figure 7. It can be clearly observed that the bead size increased when stabilized by NCh-S compared to NCh-L, which is in line with the observations made for sunflower-in-water droplets (Figure 6). Figure 7 also supports the calculated surface coverage for NCh-S (Table S2). For the three nanochitins, the interface was homogeneously covered with $\mathrm{NCh}$ nanoparticles that bent around the droplets, indicating their flexibility. A denser interfacial packing is observed for NCh-S while a looser network organization occurred with the longer NCh-L. Note that the neat and small PS spherical beads observed around the larger ones are explained by the extrusion of the St monomer through the surface and the polymerization in the water phase initiated by thermally activated AIBN with free, isolated NCh acting as nucleation sites. ${ }^{51}$

Figure $6 \mathrm{~b}$ shows that the drop size distribution was unimodal for all emulsions, and the peak width became gradually narrower as the emulsions were stabilized from $\mathrm{NCh}$ $\mathrm{S}$ to NCh-L. The microstructure of droplets obtained from NCh-S, -M, and -L is illustrated in Figure 6c-e, respectively, 

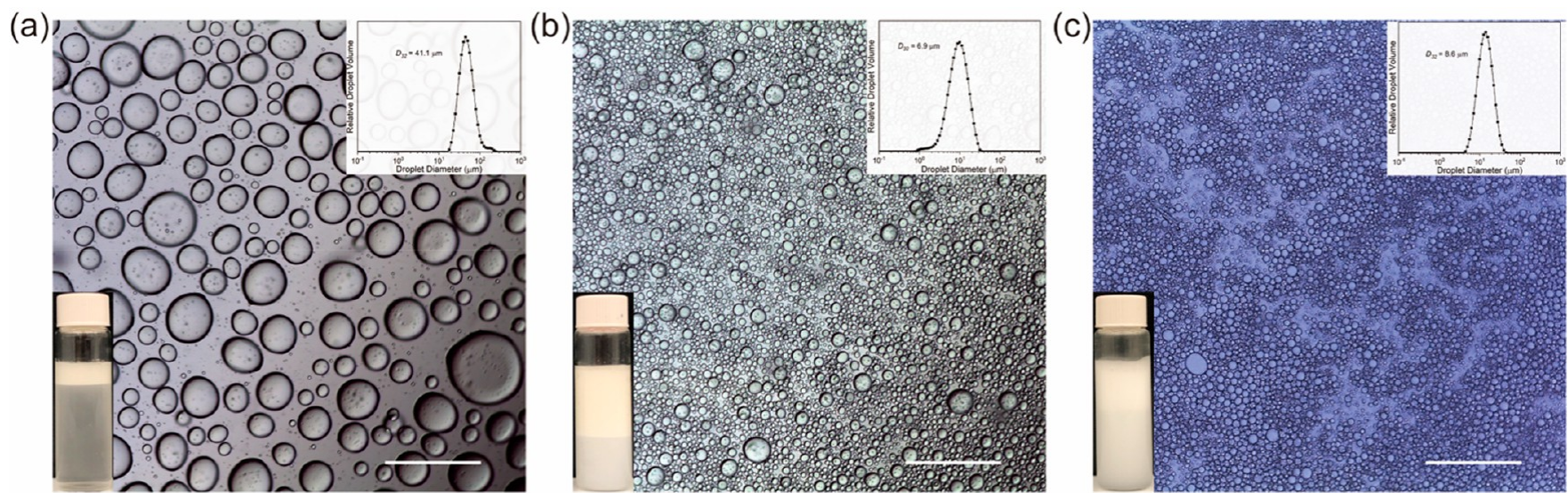

Figure 8. Optical microscope images of oil-in-water Pickering emulsions with (a) $0.001 \mathrm{wt} \%$ NCh-L concentration at 10 wt $\%$ sunflower oil, (b) $0.01 \mathrm{wt} \% \mathrm{NCh}-\mathrm{L}$ concentration at $50 \mathrm{wt} \%$ sunflower oil, and (c) $0.01 \mathrm{wt} \% \mathrm{NCh}-\mathrm{L}$ concentration at $50 \mathrm{wt} \%$ dodecane. The diameter distribution and visual appearance of the emulsions are included in each image, upper right and bottom left, respectively. The droplet diameters in (a), (b), and (c) are $41.1,6.9$, and $8.6 \mu \mathrm{m}$, respectively. The characterizations were performed after $24 \mathrm{~h}$ storage. The scale bar is $200 \mu \mathrm{m}$.

showing homogeneously distributed droplets. From merged images, red oil droplets surrounded by blue contour (dyed $\mathrm{NCh}$ ) can be clearly identified, indicating that NCh adsorbed at the oil-water interface, regardless of the axial ratio. Interestingly, a larger number of the long NCh-L nanoparticles was observed in the aqueous phase (Figures 6e and S4d), implying their restricted interfacial adsorption. One can speculate that NCh-L may be subjected to limited diffusion and mass transport from the bulk to the oil-water interface, thereby increasing the free NCh-L. The rheological measurements (Figure S7c,d) showed shear thinning behavior for all emulsions ( $n>1$, Table S1) and high storage modulus, leading to the formation of emulsion gels that facilitated stability during storage.

Interestingly, although NCh-L was present in the aqueous phase (Figures 6e and S4d), and the oil droplet size was in the micron range, no depletion flocculation occurred in the emulsions, even after storage for $>60$ days (Figure S8c), which is in stark contrast to other systems comprising nonadsorbing polymer stabilizers. ${ }^{52}$ Indeed, by using nonadsorbing CNF in CNC-stabilized Pickering emulsions, depletion flocculation occurred owing to the effect of osmotic pressure between droplets generated by $\mathrm{CNF}$ flocs dispersed in the aqueous phase. ${ }^{50} \mathrm{~A}$ main difference between $\mathrm{CNF}$ and deacetylated $\mathrm{NCh}$ is the less charged nature of the former, ${ }^{53}$ which increases the probability for floc formation. Furthermore, the nonscreened, high-surface charge of droplets also prevents interdroplet flocculation.

NCh-L was produced from one-pass microfluidization and therefore it was not subjected to intense ultrasonication before emulsion preparation. The NCh-L suspension contained fractions of relatively small chitin nanoparticles, given the sample's polydispersity (Figure 1d). It is also possible that nanochitin, especially those of larger size, disintegrated into smaller sizes during the ultrasound used for emulsification, that is, when subjected to the shear forces generated by ultrasound, due to acoustic cavitation. ${ }^{54}$ This unintended "secondary ultrasonication" of NCh-L under the conditions used for emulsion formation is illustrated in Figure S9. For comparison, $\mathrm{NCh}-\mathrm{S}$ and -M displayed no observable change in morphology (Figure S9a,b). The properties of corresponding suspensions (light scattering/turbidity), surface activity and rheological behavior were unaffected. Accordingly, the effect of the secondary ultrasonication on emulsion preparation for NCh$S$ and $-M$ was negligible. In contrast, the secondary ultrasonication did change the properties of NCh-L, as shown in Figures 2, S5b, and S9c. As shown in Figure S9c, the suspension scattered less light compared to that before ultrasonication (Figure 1d), indicating a change in nanoparticle size and better dispersion. In fact, TEM micrograph indicated that upon short-time ultrasonication, a large number of shorter nanoparticles was generated, which was beneficial for stabilizing oil droplets. Simultaneously, disintegration of nanoparticles occurred laterally, resulting in nanoparticles with smaller widths, which agrees with the possibility of loosening bonded chitin nanoparticles laterally. The increased shear viscosity, which was attributed to more nanoparticles dispersed in aqueous phase, also supported the above observations (Figure S5b). Therefore, based on these results, it is proposed that secondary disintegration applied during emulsification with NCh-L, affected their efficiency in stabilizing the oil phase, namely, size reduction of NCh-L resulted in a larger number of chitin nanoparticles at the given loading, offering a possibility to produce fine emulsions at low concentrations. Once the surface of oil droplets was sufficiently covered at the given NCh-L concentration, the effect of excess nanoparticles might be negligible.

The Pickering emulsion of low internal phase concentration ( $10 \%$ sunflower oil) produced at 0.001 wt $\%$ NCh-L is shown in Figure 8a, which shows a smaller oil droplet size $(\sim 41 \mu \mathrm{m})$ compared to that measured for NCh-S at the same concentration (see Figure 3c). For systems of higher internal phase concentration (oil fractions $=50 \%$ ) and stabilized with $0.01 \mathrm{wt} \% \mathrm{NCh}$, the emulsions presented a reduced droplet size $(\sim 7 \mu \mathrm{m})$ and narrow size distribution (Figure $8 \mathrm{~b}$ ). Furthermore, the oil droplets were well dispersed in the aqueous phase with no flocculation. The emulsions stabilized with NCh-L were stable against oiling off over 14 days, which is longer than emulsions prepared with NCh-S. The better stabilization of NCh-L is explained by the increased number density of nanoparticles: the secondary disassembly or deconstruction generated fractionation and improved interfacial coverage and stability of the oil droplets.

Further extending the system in Figure $8 \mathrm{~b}$, concentrated emulsion (oil fraction $=0.5$ or $\mathrm{WOR}=1$ ) obtained from dodecane (Equivalent Alkane Carbon Number, EACN = 10), 


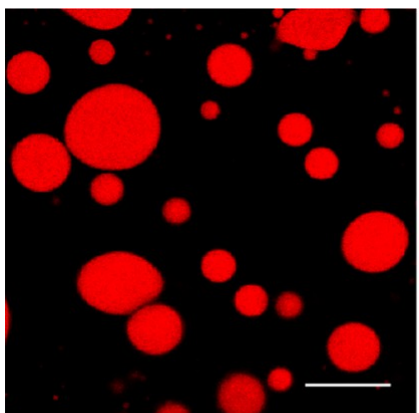

0.005 wt $\%$

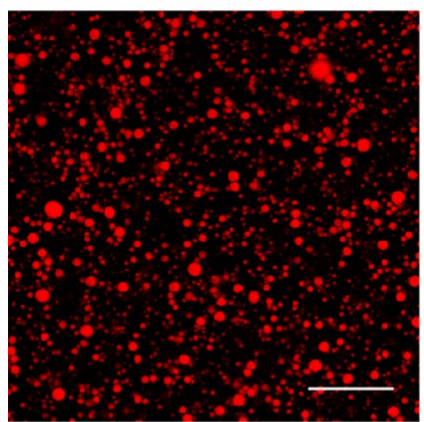

$0.2 \mathrm{wt} \%$

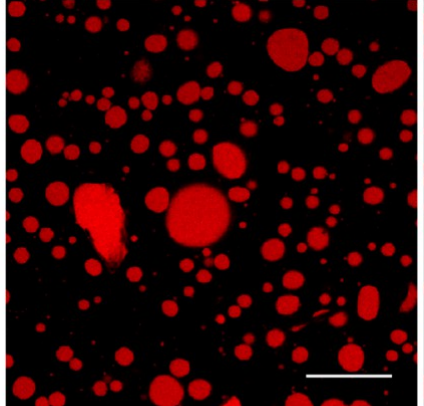

$0.01 \mathrm{wt} \%$

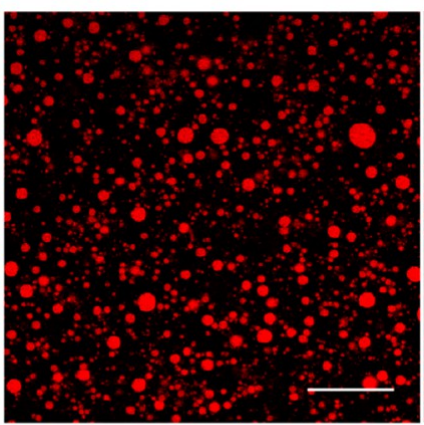

$0.3 \mathrm{wt} \%$

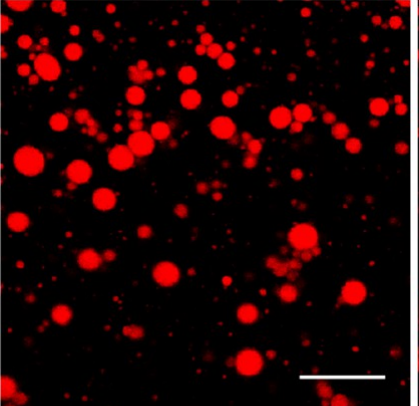

$0.05 w t \%$

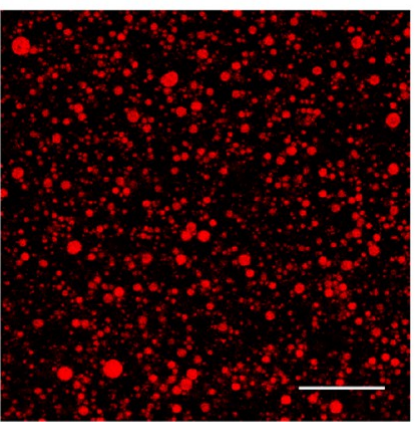

$0.5 \mathrm{wt} \%$

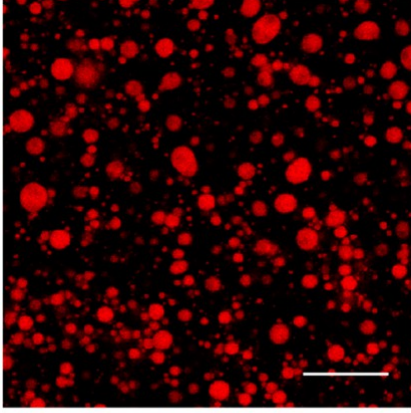

$0.1 \mathrm{wt} \%$

\section{Day-60}

Figure 9. CLSM images of NCh-S-stabilized Pickering emulsions after 60 day storage. The emulsions contained 10 wt $\%$ sunflower oil and NCh-S concentration of $0.005,0.01,0.05,0.1,0.2,0.3$, or $0.5 \mathrm{wt} \%$, as indicated. The scale bar is $20 \mu \mathrm{m}$. All the samples were stored at room temperature.

which is less polar than sunflower oil (EACN $\sim 14)$, was formulated with 0.01 wt \% NCh-L (Figure 8c). Remarkably, the formed dodecane-in-water emulsion with average droplet size of $\sim 9 \mu \mathrm{m}$, exhibited excellent stability against oiling off after $24 \mathrm{~h}$ storage. Notably, the droplet size for dodecane and sunflower oil was similar, implying a somewhat comparable interfacial coverage of NCh-L for both oils (note that the volume of dodecane, and thus the droplets surface area, was larger compared to sunflower oil due the lower density). Overall, interfacial adsorption of $\mathrm{NCh}$ on nonpolar dodecane was comparable to that on the more polar sunflower oil, supporting the concept that partial deacetylation generates random coexistence of hydrophobic and hydrophilic groups on $\mathrm{NCh}$ rods, improving their interfacial wettability.

It should be noted that compared to other Pickering systems, such as CNC-stabilized concentrated emulsion containing dodecane, ${ }^{48}$ both the stabilizer loading level as well as corresponding droplet size were much smaller for NCh$\mathrm{L}$, even if its axial ratio is much larger than that of CNC. The ability of NCh to achieve oil-in-water Pickering emulsion stable against oiling off at low concentrations, particularly for highly concentrated emulsions, is reported here for the first time and highlights the benefits of $\mathrm{NCh}$ in the formulation of multiphase system with some added advantages, namely, (i) NCh bears cationic charges, most desirable in many applications; (ii) NCh allows functional emulsions, for example, as an antimicrobial; (iii) deconstruction is greatly facilitated at low energy by simple, green deacetylation process, and (iv) use of oxidative protocols or harsh chemical treatments is avoided in the pursuit of systems with high electrostatic charges.

Long-Term Stability. Long-term stability and corresponding change in droplet size of sunflower oil-in-water Pickering emulsions stabilized by NCh-S was evaluated after storage for
21 and 60 days (Figure S10). The emulsions stabilized with 0.001 wt \% NCh-S showed extensive oil coalescence after 7 day storage (the droplet size was too large for measurement, due to obvious oiling-off on the vial wall). For other emulsions (except 0.3 and 0.5 wt \%), only creaming was observed after 60-day storage (Figure S10a, bottom). As shown in Figure $\mathrm{S} 10 \mathrm{~b}$, for creamed emulsions the oil droplets stabilized at low NCh-S concentrations ( $\leq 0.05$ wt $\%)$ coalesced into larger droplets after storage. This result can be rationalized by the low interfacial coverage at low NCh-S concentrations, as shown in Table S2. This also leads to the fact that inhibition of oil coalescence during storage depends on the nanoparticle type, composition, and formulation but in some of the cases it is a temporal effect. ${ }^{49}$ However, no oiling-off was observed in such samples (except 0.001 wt \%) at the tested time scales. The adsorbed NCh-S could resist interfacial disruption by gravity and collision, and generated electrostatic repulsion between droplets, restricting droplet collision, which competed with the total breakage of oil droplets. By increasing NCh-S to 0.1 and 0.2 wt \%, the synergy between the above effects, particularly for large surface coverages enabled by high NCh-S concentrations (Table S2), was sufficient to fully protect oil droplets to resist coalescent during long-term storage, similar to the results obtained from fresh emulsions (Figure 3).

The oil droplets kept their shape and distributed homogeneously during 60-day storage, even for emulsions with large droplets, see CLSM images in Figure 9. A clear increase in droplet size was noted for emulsions formulated with NCh-S concentration, from 0.005 to 0.05 wt \%, which is in line with light scattering results (Figure S10b). However, oil droplets with smaller size could also be observed, indicating that the oil coalescence was somehow unavoidable but at a relatively slow rate. Furthermore, once NCh-S concentration was $>0.1 \mathrm{wt} \%$, the oil droplets were stable against oil 
coalescence and flocculation, which was ascribed to better interfacial coverage by NCh-S and/or the formation of an emulsion gel. NCh-S loadings $>0.2$ wt $\%$ produced emulsion gels that were not subjected to creaming during long-term storage and displayed no changes in droplet size (Figure S13). These results confirm the stabilization property of NChstabilized Pickering emulsions after long-term storage (in the best cases, the system was stable for at least four month storage).

The storage stability of Pickering emulsions formulated with $\mathrm{NCh}$ with different axial ratios and that from NCh-S at varied internal phase fraction are displayed in Figures S8 and S11, respectively. The corresponding change in droplet size is shown in Figure S12. As shown, emulsions produced with different oil fractions at $0.5 \mathrm{wt} \% \mathrm{NCh}-\mathrm{S}$ or with $\mathrm{NCh}$ of various axial ratios (at $0.3 \mathrm{wt} \%$ concentration) were stable against creaming and oil separation after 60 day storage. In addition, the droplet size is kept nearly unchanged during the observation time. Thus, the type of emulsion gel formed explains the observations made during extended storage (Figure S13). The CLSM images in Figures S8c and S11c show that all the emulsions presented well-dispersed oil droplets with similar droplet size compared to the light scattering results obtained from corresponding fresh emulsions (Figures 6 and S6). These results directly demonstrate the excellent performance of $\mathrm{NCh}$ in the formulation of Pickering systems.

\section{CONCLUSIONS}

Stable oil-in-water Pickering emulsions were successfully prepared with nanochitins of different axial ratio (NCh-S, $-\mathrm{M}$, and -L) produced from ultrasonication and microfluidization. Irreversible stabilization of oil droplets was determined for given oil and $\mathrm{NCh}$ concentrations as well as oil (EACN) and NCh (axial ratio) types. The deacetylation process before transformation of chitin into the nanoparticles improved the interfacial wettability and enabled high electrosteric stabilization. Pickering emulsions produced from NCh-S proved to be stable against oiling off over months at a wide range of concentrations. Emulsion gels formed at high NCh-S loadings led to noncreamed, homogeneous emulsions throughout storage. NCh-M or -L showed a comparable ability as that of NCh-S to stabilize emulsions, except that larger droplets were formed with NCh-L. In this latter case, ultrasonication during emulsion preparation produced a secondary $\mathrm{NCh}$ disintegration process. This resulted in a higher emulsifying ability at low concentrations. Thus, extremely low NCh-L concentrations (down to $0.001 \mathrm{wt} \%$ ) were effective in producing small oil droplets that resisted oiling off for extended times. Concentrated emulsions with an oil fraction $=0.5($ WOR $=1)$, containing sunflower oil $($ EACN $\sim 14$ ) or nonpolar dodecane (EACN 10), were successfully produced with $0.01 \mathrm{wt} \% \mathrm{NCh}-\mathrm{L}$, resulting in fine droplets and high stability against oiling off. Our results demonstrate the tenability and versatility of nanochitin as naturally derived, biobased stabilizer, opening a route to controllably design and formulate green Pickering emulsions. The long-term goal of this research is to synergistically endow multifunctional attributes into Pickering systems with the assistance of chitin nanoparticles, which creates emulsions that exhibit antioxidant and antimicrobial capabilities simultaneously.

\section{ASSOCIATED CONTENT}

\section{Supporting Information}

The Supporting Information is available free of charge on the ACS Publications website at DOI: 10.1021/acssuschemeng.8b04023.

Additional materials are provided related to conductometric titration and SEM-EDX of partial deacetylated chitin; enlarged TEM micrographs for NCh; histogram of nanochitin ( $\mathrm{NCh}$ ) length and width; fluorescent images of simultaneous observation of $\mathrm{NCh}$ and sunflower oil in NCh-stabilized emulsions at enlarged magnification; rheological results of NCh suspensions and NCh-stabilized emulsions; visual appearance and droplet distribution of NCh-S-stabilized emulsions at different WORs; visual appearance and TEM micrographs of NCh suspensions after ultrasonication; droplet diameter, visual appearance, rheological results and CLSM images of NCh-stabilized emulsions after storage; the modeling data for rheological results; calculated surface coverage ratio of $\mathrm{NCh}$ on the oil droplets (PDF)

\section{AUTHOR INFORMATION}

\section{Corresponding Authors}

*E-mail: orlando.rojas@aalto.fi.

*E-mail: long.bai@aalto.fi.

ORCID ${ }^{\circ}$

Long Bai: 0000-0003-3356-9095

Wenchao Xiang: 0000-0003-4281-3109

Yimin Fan: 0000-0003-2764-1310

Orlando J. Rojas: 0000-0003-4036-4020

\section{Author Contributions}

The manuscript was written through contributions of all authors. All authors have given approval to the final version of the manuscript.

Notes

The authors declare no competing financial interest.

\section{ACKNOWLEDGMENTS}

We acknowledge funding support from the European Research Commission via the ERC Advanced Grant 78848 "BioElCell". This work made use of the facilities of Aalto University's Nanomicroscopy Center. Ling Wang is thanked for the help of SEM-EDX measurement.

\section{REFERENCES}

(1) McClements, D. J. Food emulsions: principles, practices, and techniques; CRC Press, 2015.

(2) Bai, L.; Huan, S.; Gu, J.; McClements, D. J. Fabrication of oil-inwater nanoemulsions by dual-channel microfluidization using natural emulsifiers: Saponins, phospholipids, proteins, and polysaccharides. Food Hydrocolloids 2016, 61, 703-711.

(3) Ramsden, W. Separation of solids in the surface-layers of solutions and 'suspensions' (observations on surface-membranes, bubbles, emulsions, and mechanical coagulation).-Preliminary account. Proc. R. Soc. London 1904, 72, 156-164.

(4) Pickering, S. U. CXCVI.-emulsions. J. Chem. Soc., Trans. 1907, 91, 2001-2021.

(5) Aveyard, R.; Binks, B. P.; Clint, J. H. Emulsions stabilised solely by colloidal particles. Adv. Colloid Interface Sci. 2003, 100, 503-546.

(6) Peddireddy, K. R.; Nicolai, T.; Benyahia, L.; Capron, I. Stabilization of water-in-water emulsions by nanorods. ACS Macro Lett. 2016, 5, 283-286. 
(7) Berton-Carabin, C. C.; Schroën, K. Pickering emulsions for food applications: background, trends, and challenges. Annu. Rev. Food Sci. Technol. 2015, 6, 263-297.

(8) Bai, L.; Greca, L. G.; Xiang, W.; Lehtonen, J.; Huan, S.; Nugroho, R. W. N.; Tardy, B. L.; Rojas, O. J. Adsorption and assembly of cellulosic and lignin colloids at oil/water interfaces. Langmuir 2019, 35, 571.

(9) Zhang, Z.; Tam, K. C.; Wang, X.; Sèbe, G. Inverse Pickering Emulsions Stabilized by Cinnamate Modified Cellulose Nanocrystals as Templates To Prepare Silica Colloidosomes. ACS Sustainable Chem. Eng. 2018, 6, 2583-2590.

(10) Qi, L.; Luo, Z.; Lu, X. Facile synthesis of starch-based nanoparticle stabilized Pickering emulsion: its $\mathrm{pH}$-responsive behavior and application for recyclable catalysis. Green Chem. 2018, 20, 15381550.

(11) Liu, F.; Tang, C.-H. Emulsifying properties of soy protein nanoparticles: influence of the protein concentration and/or emulsification process. J. Agric. Food Chem. 2014, 62, 2644-2654.

(12) Binks, B. P.; Philip, J.; Rodrigues, J. A. Inversion of silicastabilized emulsions induced by particle concentration. Langmuir 2005, 21, 3296-3302.

(13) Dickinson, E. Food emulsions and foams: stabilization by particles. Curr. Opin. Colloid Interface Sci. 2010, 15, 40-49.

(14) Bai, L.; Xiang, W.; Huan, S.; Rojas, O. J. Formulation and stabilization of concentrated edible oil-in-water emulsions based on electrostatic complexes of a food-grade cationic surfactant (ethyl lauroyl arginate) and cellulose nanocrystals. Biomacromolecules 2018, 19, 1674-1685.

(15) Ling, S.; Chen, W.; Fan, Y.; Ke, Z.; Jin, K.; Haipeng, Y.; Buehler, M. J.; Kaplan, D. L. Biopolymer nanofibrils: Structure, modeling, preparation, and applications. Pro. Polym. Sci. 2018.

(16) Duan, B.; Huang, Y.; Lu, A.; Zhang, L. Recent Advances in Chitin Based Materials Constructed via Physical Methods. Prog. Polym. Sci. 2018, 82, 1-33.

(17) Rinaudo, M. Chitin and chitosan: properties and applications. Prog. Polym. Sci. 2006, 31, 603-632.

(18) Liu, Y.; Liu, M.; Yang, S.; Luo, B.; Zhou, C. Liquid Crystalline Behaviors of Chitin Nanocrystals and their Reinforcing Effect on Natural Rubber. ACS Sustainable Chem. Eng. 2018, 6, 325-336.

(19) Duan, B.; Zheng, X.; Xia, Z.; Fan, X.; Guo, L.; Liu, J.; Wang, Y.; Ye, Q.; Zhang, L. Highly Biocompatible Nanofibrous Microspheres Self-Assembled from Chitin in $\mathrm{NaOH} / \mathrm{Urea}$ Aqueous Solution as Cell Carriers. Angew. Chem., Int. Ed. 2015, 54, 5152-5156.

(20) Shen, X.; Shamshina, J. L.; Berton, P.; Bandomir, J.; Wang, H.; Gurau, G.; Rogers, R. D. Comparison of hydrogels prepared with ionic-liquid-isolated vs commercial chitin and cellulose. ACS Sustainable Chem. Eng. 2016, 4, 471-480.

(21) Salaberria, A. M.; Diaz, R. H.; Labidi, J.; Fernandes, S. C. Role of chitin nanocrystals and nanofibers on physical, mechanical and functional properties in thermoplastic starch films. Food Hydrocolloids 2015, 46, 93-102.

(22) Zeng, J.-B.; He, Y.-S.; Li, S.-L.; Wang, Y.-Z. Chitin whiskers: An overview. Biomacromolecules 2012, 13, 1-11.

(23) Fan, Y.; Saito, T.; Isogai, A. Preparation of chitin nanofibers from squid pen $\beta$-chitin by simple mechanical treatment under acid conditions. Biomacromolecules 2008, 9, 1919-1923.

(24) Salaberria, A. M.; Labidi, J.; Fernandes, S. C. Chitin nanocrystals and nanofibers as nano-sized fillers into thermoplastic starch-based biocomposites processed by melt-mixing. Chem. Eng. J. 2014, 256, 356-364.

(25) Tzoumaki, M. V.; Moschakis, T.; Scholten, E.; Biliaderis, C. G. In vitro lipid digestion of chitin nanocrystal stabilized o/w emulsions. Food Funct. 2013, 4, 121-129.

(26) Tzoumaki, M. V.; Moschakis, T.; Kiosseoglou, V.; Biliaderis, C. G. Oil-in-water emulsions stabilized by chitin nanocrystal particles. Food Hydrocolloids 2011, 25, 1521-1529.

(27) Wang, X.; Liang, K.; Tian, Y.; Ji, Y. A facile and green emulsion casting method to prepare chitin nanocrystal reinforced citrate-based bioelastomer. Carbohydr. Polym. 2017, 157, 620-628.
(28) Tian, Y.; Liang, K.; Wang, X.; Ji, Y. Fabrication of Nanocomposite Bioelastomer Porous Scaffold Based on Chitin Nanocrystal Supported Emulsion-Freeze-Casting. ACS Sustainable Chem. Eng. 2017, 5, 3305-3313.

(29) Barkhordari, M. R.; Fathi, M. Production and characterization of chitin nanocrystals from prawn shell and their application for stabilization of Pickering emulsions. Food Hydrocolloids 2018, 82, $338-345$.

(30) Bai, L.; Huan, S.; Li, Z.; McClements, D. J. Comparison of emulsifying properties of food-grade polysaccharides in oil-in-water emulsions: Gum arabic, beet pectin, and corn fiber gum. Food Hydrocolloids 2017, 66, 144-153.

(31) Liu, L.; Wang, R.; Yu, J.; Jiang, J.; Zheng, K.; Hu, L.; Wang, Z.; Fan, Y. Robust self-standing chitin nanofiber/nanowhisker hydrogels with designed surface charges and ultralow mass content via gas phase coagulation. Biomacromolecules 2016, 17, 3773-3781.

(32) Fan, Y.; Saito, T.; Isogai, A. Individual chitin nano-whiskers prepared from partially deacetylated $\alpha$-chitin by fibril surface cationization. Carbohydr. Polym. 2010, 79, 1046-1051.

(33) Larbi, F.; García, A.; del Valle, L. J.; Hamou, A.; Puiggalí, J.; Belgacem, N.; Bras, J. Comparison of nanocrystals and nanofibers produced from shrimp shell $\alpha$-chitin: From energy production to material cytotoxicity and Pickering emulsion properties. Carbohydr. Polym. 2018, 196, 385-397.

(34) Zhang, Y.; Chen, Z.; Bian, W.; Feng, L.; Wu, Z.; Wang, P.; Zeng, X.; Wu, T. Stabilizing oil-in-water emulsions with regenerated chitin nanofibers. Food Chem. 2015, 183, 115-121.

(35) Huang, Y.; Yang, J.; Chen, L.; Zhang, L. Chitin Nanofibrils to Stabilize Long-life Pickering Foams and Their Application for Lightweight Porous Materials. ACS Sustainable Chem. Eng. 2018, 6, 10552-10561.

(36) Pang, K.; Ding, B.; Liu, X.; Wu, H.; Duan, Y.; Zhang, J. Highyield preparation of a zwitterionically charged chitin nanofiber and its application in a doubly $\mathrm{pH}$-responsive Pickering emulsion. Green Chem. 2017, 19, 3665-3670.

(37) Salaberria, A. M.; Labidi, J.; Fernandes, S. C. Different routes to turn chitin into stunning nano-objects. Eur. Polym. J. 2015, 68, 503515 .

(38) Kalashnikova, I.; Bizot, H.; Bertoncini, P.; Cathala, B.; Capron, I. Cellulosic nanorods of various aspect ratios for oil in water Pickering emulsions. Soft Matter 2013, 9, 952-959.

(39) Liu, L.; Borghei, M.; Wang, Z.; Xu, J.; Fan, Y.; Rojas, O. J. SaltInduced Colloidal Destabilization, Separation, Drying, and Redispersion in Aqueous Phase of Cationic and Anionic Nanochitins. J. Agric. Food Chem. 2018, 66, 9189-9198.

(40) Liu, L.; Bai, L.; Tripathi, A.; Yu, J.; Wang, Z.; Borghei, M.; Fan, Y.; Rojas, O. J. High Axial Ratio Nanochitins for Ultra-Strong and Shape-Recoverable Hydrogels and Cryogels via Ice Templating. ACS Nano 2019, DOI: 10.1021/acsnano.8b07235.

(41) Riehle, F.; Hoenders, D.; Guo, J.; Eckert, A.; Ifuku, S.; Walther, A. Sustainable Chitin Nanofibrils Provide Outstanding FlameRetardant Nanopapers. Biomacromolecules 2019, 20, 1098.

(42) Schneider, C. A.; Rasband, W. S.; Eliceiri, K. W. NIH Image to ImageJ: 25 years of image analysis. Nat. Methods 2012, 9, 671-675.

(43) Li, Q.; Wang, Y.; Wu, Y.; He, K.; Li, Y.; Luo, X.; Li, B.; Wang, C.; Liu, S. Flexible Cellulose Nanofibrils as Novel Pickering Stabilizers: The Emulsifying Property and Packing Behavior. Food Hydrocolloids 2019, 88, 180-189.

(44) Al-Sawalmih, A.; Li, C.; Siegel, S.; Fabritius, H.; Yi, S.; Raabe, D.; Fratzl, P.; Paris, O. Microtexture and chitin/calcite orientation relationship in the mineralized exoskeleton of the American lobster. Adv. Funct. Mater. 2008, 18, 3307-3314.

(45) Bai, L.; McClements, D. J. Development of microfluidization methods for efficient production of concentrated nanoemulsions: Comparison of single-and dual-channel microfluidizers. J. Colloid Interface Sci. 2016, 466, 206-212.

(46) Bai, L.; McClements, D. J. Formation and stabilization of nanoemulsions using biosurfactants: Rhamnolipids. J. Colloid Interface Sci. 2016, 479, 71-79. 
(47) Toivonen, M. S.; Onelli, O. D.; Jacucci, G.; Lovikka, V.; Rojas, O. J.; Ikkala, O.; Vignolini, S. Anomalous-Diffusion-Assisted Brightness in White Cellulose Nanofibril Membranes. Adv. Mater. 2018, 30, 1704050.

(48) Hu, Z.; Ballinger, S.; Pelton, R.; Cranston, E. D. Surfactantenhanced cellulose nanocrystal Pickering emulsions. J. Colloid Interface Sci. 2015, 439, 139-148.

(49) Kalashnikova, I.; Bizot, H.; Cathala, B.; Capron, I. New Pickering emulsions stabilized by bacterial cellulose nanocrystals. Langmuir 2011, 27, 7471-7479.

(50) Bai, L.; Huan, S.; Xiang, W.; Rojas, O. J. Pickering emulsions by combining cellulose nanofibrils and nanocrystals: Phase behavior and depletion stabilization. Green Chem. 2018, 20, 1571-1582.

(51) Werner, A.; Schmitt, V.; Sèbe, G.; Héroguez, V. Synthesis of surfactant-free micro-and nanolatexes from Pickering emulsions stabilized by acetylated cellulose nanocrystals. Polym. Chem. 2017, $8,6064-6072$.

(52) Bai, L.; Liu, F.; Xu, X.; Huan, S.; Gu, J.; McClements, D. J. Impact of polysaccharide molecular characteristics on viscosity enhancement and depletion flocculation. J. Food Eng. 2017, 207, $35-45$.

(53) Huan, S.; Yokota, S.; Bai, L.; Ago, M.; Borghei, M.; Kondo, T.; Rojas, O. J. Formulation and Composition Effects in Phase Transitions of Emulsions Costabilized by Cellulose Nanofibrils and an Ionic Surfactant. Biomacromolecules 2017, 18, 4393-4404.

(54) Peshkovsky, A. S.; Peshkovsky, S. L.; Bystryak, S. Scalable highpower ultrasonic technology for the production of translucent nanoemulsions. Chem. Eng. Process. 2013, 69, 77-82. 